\title{
Multidimensional item response theory and the Brief Electricity and Magnetism Assessment
}

\author{
John Hansen® and John Stewart๑* \\ Department of Physics and Astronomy, West Virginia University, Morgantown, West Virginia 26506, USA
}

(Received 4 August 2021; accepted 2 November 2021; published 30 November 2021)

\begin{abstract}
This work is the fourth of a series of papers applying multidimensional item response theory (MIRT) to widely used physics conceptual assessments. This study applies MIRT analysis using both exploratory and confirmatory methods to the Brief Electricity and Magnetism Assessment (BEMA) to explore the assessment's structure and to determine a well-fitting model of student knowledge measured by the assessment. These methods were used to investigate a large dataset $(N=9666)$ from a research university in the United States. Exploratory analysis showed that a five-factor model had the best fit statistics; the items with the highest loadings in four of the five factors were items in the same item block. Confirmatory MIRT analysis fit a theoretical model developed from expert solutions to the instrument and identified two models with superior model fit: a principle model and a topical model. The principle model consisted of 28 principles, fundamental reasoning steps needed to solve items in the instrument; this was more principles than any of the models in the previous confirmatory MIRT studies of the Force Concept Inventory, the Force and Motion Conceptual Evaluation, and the Conceptual Survey of Electricity and Magnetism. A second model, the topical model, consisted of five general subtopics of electromagnetism. Both the principle and the topical model had excellent fit statistics; however, unlike the other conceptual instruments studied, the topical model had better fit statistics. The five topical divisions were explored as possible subscales; however, none of these subscales had a Cronbach's $\alpha$ of 0.7 , the minimum value for required low-stakes testing.
\end{abstract}

DOI: 10.1103/PhysRevPhysEducRes.17.020139

\section{INTRODUCTION}

The Brief Electricity and Magnetism Assessment (BEMA) was developed to measure students' qualitative understanding of basic concepts in electricity and magnetism [1,2]. The BEMA and the Conceptual Survey of Electricity and Magnetism (CSEM) [3] have been used in the majority of physics education research (PER) studies of conceptual understanding of electricity and magnetism. Both were developed after Halloun and Hestenes demonstrated that students leave traditional physics classes with little change in their conceptual understanding [4]. This observation lead to the development of the broadly applied Force Concept Inventory (FCI) [5] which measured conceptual understanding of Newtonian mechanics. Using the FCI, Hake demonstrated that the failure of traditional instruction to foster conceptual learning gains was common to physics classes at many institutions [6]. The introduction of the FCI, CSEM, and BEMA as well as the Force and Motion Conceptual Evaluation (FMCE) [7] began an

\footnotetext{
*jcstewart1@mail.wvu.edu
}

Published by the American Physical Society under the terms of the Creative Commons Attribution 4.0 International license. Further distribution of this work must maintain attribution to the author(s) and the published article's title, journal citation, and DOI. extensive research strand in PER studying student understanding with multiple-choice conceptual instruments [8].

\section{A. The Brief Electricity and Magnetism Assessment}

The BEMA is a 31-item multiple-choice instrument that covers electricity and magnetism topics [1,2]. It includes items covering electrostatics, electric potential, magnetostatics, and magnetic induction. This study used the version available from PhysPort [9]. Unlike the CSEM, the BEMA also includes 6 items involving electric circuits and 4 items asking the students to select responses involving quantitative formulas. The items present students with a variable number of possible responses with some items using up to 10 responses. Most responses include either a "none of the above" response or a response that is zero; these types of responses have been shown to cause psychometric problems in other instruments [10].

The instrument contains multiple "item blocks" where multiple items refer to a common item stem or a common description of the physics system. Items $\{1,2,3\},\{4,5\}$, $\{8,9\},\{14,15,16\},\{21,22\},\{26,27\}$, and $\{28,29\}$ are blocked. Multiple studies have shown that the practice of item blocking can generate correlations between the blocked items that make them difficult to interpret [11-13].

The version of the BEMA at PhysPort [9] suggests a scoring rubric which accounts for some of the relations 
between the items. Item 3 is to be graded as correct if it is answered correctly based on the response to item 2 (both involve the forces on two point charges). Item 16 is to be graded as correct if it is consistent with item 14 and if the answer to item 15 is zero. Items 14 to 16 ask about the potential difference between different points in a uniform electric field. Items 28 and 29 are to be graded together; the student receives one point if both are correct, zero otherwise. By grading items 28 and 29 as a group, the total score on the instrument is reduced from 31 to 30 .

The BEMA contains 5 items which are nearly identical to items on the CSEM only differing by the number of responses. Items 1, 2, and 3 are very similar to CSEM items 3,4 , and 5. These items are blocked in both instruments. BEMA items 30 and 31 are likewise similar to CSEM items 31 and 32, again differing by the number of responses.

\section{B. Research questions}

This work is the fourth of a series of papers applying multidimensional item response theory (MIRT) to widely used physics conceptual assessments. As in the prior work, MIRT will be applied both as an exploratory method and as a confirmatory method by constraining the MIRT models to a theoretical model developed from expert solutions.

This study seeks to answer the following research questions:

RQ1: What relations between BEMA items are identified by exploratory analyses? What do these relations imply for the interpretation of the results of applying the BEMA?

RQ2: What is the model of student knowledge measured by the BEMA identified by constrained MIRT? What insights can this model provide into the structure of the instrument?

RQ3: How is the model of the BEMA related to the models of other conceptual inventories?

\section{Item response theory}

Item response theory (IRT) represents a rich set of statistical models that describe the probability a student selects a certain response in a multiple-choice instrument. Many IRT models have been used to explore physics conceptual inventories: the Rasch model [14-16], the 2parameter logistic (2PL) [17-20], the three-parameter logistic (3PL) [19,21], the nested-logit model [22], the nominal model [23], and MIRT [11-13,24]. The statistical properties of IRT models are reviewed in Sec. II B.

\section{Prior constrained MIRT studies}

Multidimensional IRT was applied as both an exploratory and confirmatory analyses method to popular physics conceptual inventories. These studies will be referenced as studies 1, 2, and 3 in this work.

\section{Study $1-F C I$}

The use of constrained MIRT was first introduced by Stewart and Zabriskie to examine the FCI [11]. The general structure of the instrument was investigated using correlation analysis, partial correlation analysis, and exploratory factor analysis (EFA) as exploratory methods. These analyses showed that a substantial part of the factor structure and partial correlation structure of the FCI could be explained by the practice of blocking items into item groups all referring to a common stem or where later items in the group directly referenced prior items. This study then applied MIRT as a confirmatory method constraining the parameter matrix to a theoretical model of the principles needed to solve each item. This model was developed from expert solutions. Principles are fundamental reasoning steps in the solution of the item. Constrained MIRT was then used to explore theoretically motivated modifications to the initial model to identify the best-fitting model. This model revealed that there were four groups of isomorphic items requiring very similar solution structure: items $\{4,15,16,28\},\{5,18\},\{6,7\}$, and $\{17,25\}$. These isomorphic items explained the factor structure not explained by the item blocks. The best-fitting MIRT model had substantially superior fit statistics than the original model of the FCI proposed by its authors.

\section{Study 2-FMCE}

The same methods as in study 1 were then applied to the FMCE by Yang et al. [13]. The FMCE makes much heavier use of blocking than the FCI, CSEM, or BEMA with all but one item included in an item block. Correlation analysis and MIRT EFA showed that these item blocks and combinations of the item blocks explained much of the structure of the instrument. Confirmatory MIRT was then used to develop a best-fitting model which showed the items in the item blocks were generally isomorphic. As such, it was impossible to determine if the similar solution structure or the practice of blocking resulted in these items being identified in the same factors by EFA. The confirmatory analysis was then used to show that the FMCE covered far fewer principles than the FCI and that the principles covered were used differently with the FMCE containing many items using a single principle while the FCI generally used items mixing a number of principles.

\section{Study 3-CSEM}

Zabriskie and Stewart applied MIRT to two CSEM datasets drawn from different institutions [12]. Study 3 identified 3 isomorphic item groups: items $\{6,8\},\{16,17\}$, and $\{21,27\}$. These isomorphic groups were less important to the exploratory factor structure with only $\{21,27\}$ loading strongly on the same factor. This work also fit a general model of the instrument using the overall categories: mechanics, electrostatics, electric potential, 
magnetostatics, magnetic induction, and superposition. Like the FCI, this general model was not as well fitting as the best-fitting constrained model; however, unlike the mechanics instruments, some fit statistics suggested the general model was superior. The best-fitting models extracted for the two institutions were very similar. Model parameters for the different institutions were different, but still related. This suggests the best-fitting models extracted may have some generality.

These works have been productively employed by other studies because they produced a detailed mapping of the concepts measured by the instrument and each demonstrated the central role of the practice of blocking items in determining the factor structure of the instrument [23,25-27].

\section{E. Prior studies of the BEMA}

The BEMA was introduced in 1997 [2] and has been used in several studies as an assessment to measure gains in electricity and magnetism conceptual knowledge [28-30]. A study conducted by Ding et al. [31] explored the reliability of the BEMA as an assessment tool examining the reliability of the instrument as a whole and of the individual items. The study looked at five statistics: item difficulty index (the score of each item), item discrimination index (a measure of how well an item discriminates between high-ability and low-ability students), point biserial coefficient (a correlation between a student's score on an individual item and their score on the entire test), the Kuder-Richardson reliability index (a measurement of a test's self-consistency) and Ferguson's delta (a measurement of the discrimination of an entire test). Each statistic indicated that the BEMA was a reliable instrument with sufficient discrimination between high-ability and lowability students. A later study by Ding [32] used Rasch theory to test the construct validity of the BEMA, and found that the BEMA does measure a unidimensional construct even though the items cover a broad range of topics in electricity and magnetism.

Kohlmyer et al. [29] used the BEMA to test the knowledge level of students in two different introductory electricity and magnetism courses: a traditional electricity and magnetism course and the second semester of the Matter and Interactions (MI) curriculum [33]. Students enrolled in the MI course had significantly higher post-test scores than the students enrolled in the traditional electricity and magnetism course at each of the four institutions studied.

Ding [16], using a dataset that was collected from students in parallel traditional and MI electromagnetism courses at the same institution, found five BEMA items with different averages in the two courses; two were higher in the MI course and three were higher in the traditional electricity and magnetism course. BEMA items 9 and 17 were also shown to be problematic because of low discrimination. Item 9 asks about current flow in an ionic channel and requires an answer with mathematical formula unlike most items in the instrument. Item 17 tests the electric potential in an open circuit.

A recent study by Xiao et al. [19] found that some conceptual instruments, including the BEMA and CSEM, could be shortened without diminishing the validity and reliability of assessment. This was done using item response theory. The latent constructs of student learning in electricity and magnetism that are measured by the BEMA were shown to be measured with similar reliability by a shortened BEMA assessment.

\section{F. Studies comparing the BEMA and the CSEM}

Xiao et al. [19] also showed that student scores on the BEMA and CSEM can be compared after linking the assessment scales and appropriately transforming them. This supports prior work done by Pollock [34]. Pollock compared the CSEM and BEMA and found them to be fairly equally effective in assessing conceptual understanding [34]; however, the instruments have somewhat different coverage. Eaton et al. [20] used item response theory and classical test theory (CTT) on the BEMA and CSEM to show that the assessments were nearly equal in overall difficulty. Any differences found between the two tests were minimal and potentially caused by differences in the test samples. The circuit questions on the BEMA were poorly correlated with other concept areas on the assessment.

Some of the differences in coverage of the BEMA and CSEM were evident in an EFA comparing the BEMA and the CSEM by Eaton et al. [35]. They concluded the two instruments cover the nearly the same conceptual content, with the exception of a few factors. The CSEM had an EFA model of six factors while the BEMA had a five-factor model. This study did not use all BEMA items, removing several items due to low Kaiser-Meyer-Olkin test values which measure how well a sample loads onto different factors.

\section{G. The structure of knowledge}

The current work will build a detailed model of the BEMA involving 50 principles of electromagnetic theory. This model shares many features with earlier models of physics problem solving constructed using the paradigm of cognitive research introduced by Simon and Newell [36]. This paradigm dominated research into problem solving for 30 years and is reviewed by Ohlsson [37]. The paradigm built exceptionally detailed, computationally functional models of the problem solving process. These models could then be run on computers to reproduce the problemsolving sequence of participants. The technique was used to understand expert-novice differences in problem solving in physics and many other fields [38,39]. This paradigm ultimately lost favor because it was difficult to explore 
general features of complex problem solving; however, in PER it is often the goal to understand specific features of the physics problem-solving process. As such, the detailed models produced by this method may be productive. Reif and Heller also produced a fine-grained model of physics problem solving, but this model did not meet the test of being computationally functional [40]. These models involved identifying the fundamental transformations, called principles, needed to navigate the problem space. These principles are closely related to the principles identified in the theoretical MIRT model; the MIRT principles take their name from these earlier works.

\section{METHODS}

\section{A. Sample}

The sample for this study was collected at a large western land-grant university in the United States serving 34000 student. Fifty percent of the undergraduate student population had ACT scores in the range 25 to 30 . The demographic composition of the general undergraduate population was $67 \%$ White, $12 \%$ Hispanic, $6 \%$ Asian, $6 \%$ two or more races, $6 \%$ International, $2 \%$ Black with other races less than $1 \%$ [41].

The aggregate dataset was drawn from 22 semesters of an introductory, calculus-based, electricity and magnetism class. It contains $9666 \mathrm{BEMA}$ post-test records. Any record that contained one or more missing responses was removed, as well as records that had suspicious response patterns, e.g., "A" repeated or "ABCDE" repeated.

\section{B. Item response theory}

Item response theory encompasses a broad collection of statistical models of the response patterns to multiplechoice instruments. These models estimate the probability of either selecting the correct response or each response in terms of a latent student-level trait called the ability. This latent trait represents the general facility of each student with the material tested by the instrument. Unidimensional IRT, estimating a single latent ability, has been used in many PER studies of the FCI, FMCE, and CSEM $[14,17,21,42-47]$. These studies are summarized in detail for the individual instruments in studies 1 to 3 .

Multidimensional IRT is a generalization of unidimensional IRT which estimates multiple latent abilities for each student. It was used as both an exploratory and confirmatory method in studies 1 to 3. MIRT was also used by Scott and Schumayer [24] to perform an exploratory factor analysis of the FCI. MIRT provided similar, but not identical results, to an earlier work on the same dataset using traditional factor analysis [48].

An exploratory analysis allows the model to be deduced from the data without the input of a theoretical model. A confirmatory analysis begins with a theoretical model and seeks to determine how well a set of data is described by the model. Studies 1 to 3 and 50 years of social science research $[49,50]$ argue that purely exploratory analyses are susceptible to misinterpreting random fluctuations in the data as real effects.

MIRT estimates the probability $\pi_{i j}$ that student $i$ will answer correctly on item $j$. For each item, MIRT estimates a parameter $d_{j}$ related to the overall difficulty of the problem. Items with larger $d_{j}$ are answered correctly more often. More difficult problems have smaller $d_{j}$, easier problems larger $d_{j}$. MIRT also estimates $K$ discrimination parameters $a_{j k}$ for each item and $K$ ability traits $\theta_{i k}$ for each student. The discrimination and the ability can be written as $K$ element vectors, $\boldsymbol{a}_{j}$ and $\boldsymbol{\theta}_{i}$. The MIRT probability model is given by Eq. (1).

$$
\pi_{i j}=\frac{\exp \left[\boldsymbol{a}_{j} \cdot \boldsymbol{\theta}_{i}+d_{j}\right]}{1+\exp \left[\boldsymbol{a}_{j} \cdot \boldsymbol{\theta}_{i}+d_{j}\right]} .
$$

Additional insight can be gained in the functioning of this model by calculating the log odds, the logarithm of the odds. The odds a student $i$ selects the correct answer on item $j$ is defined as odds $s_{i j}=\pi_{i j} /\left(1-\pi_{i j}\right)$. The log odds is then a linear equation with independent variable $\theta_{i}$, slope $a_{j}$, and intercept $d_{j}$ as shown in Eq. (2). It is the following which is fit when performing a logistic regression:

$$
\ln \left(\operatorname{odds}_{i j}\right)=\ln \left(\frac{\pi_{i j}}{1-\pi_{i j}}\right)=d_{j}+\boldsymbol{a}_{j} \cdot \boldsymbol{\theta}_{i}
$$

Some qualitative understanding of the features of the probability function are helpful when interpreting the MIRT models. For this discussion, consider a model with one discrimination parameter $(K=1)$. If $a>0$, the probability curve has the characteristic $S$ shape shown in Fig. 1. The figure shows the probability curve drawn with $d=0$ and $a=1$. With this choice of parameters, the probability of answering correctly is 0.5 at $\theta=0$. In general, the probability of answering correctly is 0.5 at $\theta_{1 / 2}$ which occurs when the argument of the exponential is zero: $\theta_{1 / 2}=-d_{j} / a_{j}$; therefore, a combination of $a_{j}$ and $d_{j}$ determine the ability at which a student has a $50 \%$ chance of answering the problem correctly. The slope of the probability at $\theta_{1 / 2}$ is $a_{j} / 4$; therefore, the discrimination $a_{j}$ is related to how fast the probability is increasing when the students have a 50\% chance of answering correctly. If $a_{j}$ is larger, the transition from low probability to high probability is faster, the item discriminates between low and high ability students more strongly. If $a_{j}=0$, the probability curve is flat, low and high ability students have equal chances of answering correctly, a characteristic of a problematic item. More problematic are items with $a_{j}<0$; for these items the $S$ curve inverts and students with low ability have a higher probability of getting the item correct than students with high ability. 


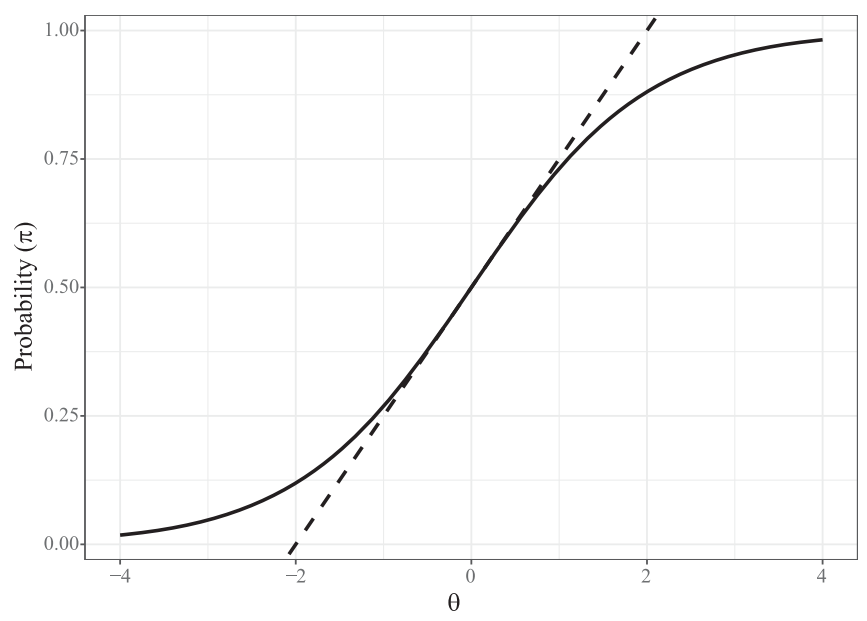

FIG. 1. Probability of selecting the correct response, $\pi(\theta)$, versus ability $\theta$ using $d=0$ and $a=1$. The dashed line represents the slope at $\theta=0$ and has slope $a / 4=0.25$.

The MIRT models were fit using the "mirt" package [51] which is part of the R software system [52]. Models were fit using the Metropolis-Hastings Robbins-Monro (MHRM) algorithm [53] which uses stochastic methods to maximize the likelihood function. Maximum likelihood estimation does not require the assumption of an underlying normal distribution.

\section{Model fit statistics}

The parameters in a MIRT model are estimated using maximum likelihood (ML) methods where the parameters are selected to make the observed response pattern the most probable using Eq. (1). Maximum likelihood methods calculate the likelihood function $L$, the probability the observed response pattern occurred given the MIRT probability model and a set of parameters. The parameters are modified until $L$ is maximized. A broad collection of model fit statistics have been developed to characterize and compare ML models. This study reports Akaike information criterion (AIC), the Bayesian information criterion (BIC), the root mean square error of approximation (RMSEA), the comparative fit index (CFI), and the Tucker-Lewis index (TLI).

AIC [Eq. (3)] and BIC [Eq. (4)] measure the relative information lost between the model fit and the true model; better fitting models lose less information and thus minimize AIC and BIC. Both penalize for the addition of parameters with BIC penalizing additional parameters more strongly:

$$
\begin{gathered}
\mathrm{AIC}=2 k-2 \ln (L), \\
\mathrm{BIC}=k \ln (n)-2 \ln (L),
\end{gathered}
$$

where $k$ is the number of parameters estimated and $n$ is the sample size. Both AIC and BIC depend on the logarithm of the likelihood, so small changes in either measure produce large changes in likelihood. Raftery provided criteria for the effect size of differences in BIC: $\triangle$ BIC $\leq 2$ as "weak," $2<\Delta \mathrm{BIC} \leq 6$ as "positive," $6<\Delta \mathrm{BIC} \leq 10$ as "strong," and $\triangle \mathrm{BIC}>10$ as "very strong" [54]. The definition of AIC and BIC are very similar; therefore, this work also adopts Raftery's convention for AIC.

RMSEA, CFI, and TLI are measures of model fit or misfit derived from the chi-squared $\left(\chi^{2}\right)$ statistic. For a $N$ item dichotomously scored instrument, there are $C=2^{N}$ possible response sequences. To calculate chi squared, the probability of each possible response sequence, $P_{c}$, is compared to the observed frequency of the sequence, $O_{c}$, $\chi^{2}=n \sum_{0}^{C}\left(O_{c}-P_{c}\right) / O_{c}$, where $n$ is the number of observations. For the BEMA with $N=31$ items and for most multiple-choice instruments of reasonable length, it would require an enormous amount of data to estimate $\chi^{2}$ accurately. As such, MIRT uses an approximation to $\chi^{2}$ called $M_{2}$ to approximate $\chi^{2}[55,56]$.

RMSEA [Eq. (5)] characterizes badness of model fit on a scale of 0 to 1 using $\chi^{2}$ normalized by the number of degrees of freedom (dof) [57]; models with larger RMSEA represent worse fitting models. RMSEA less than 0.05 represents good model fit; RMSEA above 0.10 represents poor model fit [58]:

$$
\operatorname{RMSEA}=\sqrt{\frac{\left(\chi^{2} / \mathrm{dof}\right)-1}{n-1} .}
$$

CFI [Eq. (6)] and TLI are incremental goodness-of-fit statistics which characterize how much the model differs from a null model [57]. The null model used by MIRT constrains the discrimination matrix to zero, $\vec{a}_{j}=0$, and fits the model containing only $d_{j}$. CFI and TLI values above 0.95 represent good model fit [59]:

$$
\mathrm{CFI}=1-\frac{\chi^{2}-\operatorname{dof}}{\chi_{\text {null }}^{2}-\operatorname{dof}_{\text {null }}} .
$$

The equation for TLI contains a slightly modified combination of the null and fitted models.

$\mathrm{Hu}$ and Bentler recommend using multiple fit statistics to compare models [59]. As such, a superior model has AIC and BIC at least 20 lower than other models, RMSEA near zero, and CFI and TLI near one.

The relation of RMSEA, CFI, and TLI to the number of parameters fit is complicated. All three statistics involve the ratio of an effective chi-squared statistic to the number of degrees of freedom. As more parameters are fit, generally $\chi^{2}$ decreases, but the number of degrees of freedom decreases. Eventually, the decrease in $\chi^{2}$ is not enough to compensate for the decrease in the degrees of freedom and the statistics begin to increase as the models become sufficiently complex. 


\section{RESULTS}

The BEMA was first examined with two exploratory analyses: correlation analysis and exploratory factor analysis. The instrument was then examined with a confirmatory analysis fitting a model based on expert solutions to the instrument.

\section{A. Exploratory analyses}

The BEMA was first examined using the correlation and partial correlation matrices. The correlation matrix is presented in Fig. 2. The partial correlation matrix, which corrects for correlations resulting from overall BEMA scores, is shown in Fig. 3. The partial correlation matrix shows five groups of items that are substantially positively correlated after correcting for overall BEMA score: $\{1,2,3\},\{4,5\},\{14,16\},\{21,22\}$, and $\{28,29\}$. All groups are part of item blocks. Item 15 is not present in the $\{14,16\}$ group. This group of items asks about the potential difference between various points in a uniform electric field. Item 15 asks about the potential difference along an equipotential unlike the other two items.

An EFA was performed using MIRT. To use MIRT as an exploratory method, the discrimination matrix $\boldsymbol{a}_{j}$ is allowed to vary freely. MIRT was used to extract from 1 to 10 factors; the fit statistics for each model are shown in Table I. Consistent with studies 1 to 3 , the fit statistics do not clearly identify a single best-fitting model. The six-factor model minimizes AIC and BIC while the five-factor model minimizes RMSEA, and maximizes CFI and TLI. The relatively poor RMSEA, CFI, and TLI of the six-factor model strongly indicates the five-factor model is the superior model. The factor structure of the five-factor model is shown in Table II. As in prior studies, the factor structure is dominated by the blocked items; block items form the highest loadings on factors 1 to 4 . This is consistent with the correlation analysis which shows only blocked items are more correlated with each other than with the total instrument score. This supports the work of Eaton et al. [35] who also reported a five-factor model as optimal.

\section{B. Confirmatory analyses}

For a confirmatory analysis, one first develops a theoretical model and then determines how well the data fit the model. One can also propose a small number of theoretically motivated modifications to the model. Ideally, these should be proposed before the model is initially fit. By constraining the analysis to a theoretical model, confirmatory methods are less likely to erroneously identify structure resulting from random effects.

\section{Theoretical framework}

A model of the knowledge structure measured by the BEMA is shown in Table III. This model was developed in the same way that models of knowledge structure were developed in studies 1,2, and 3. Content experts including members of the research team and instructors of introductory, calculus-based physics courses at the institution where the analysis was performed were asked to complete the BEMA and write the reasoning used to solve each problem. These responses were decomposed to the sentence or

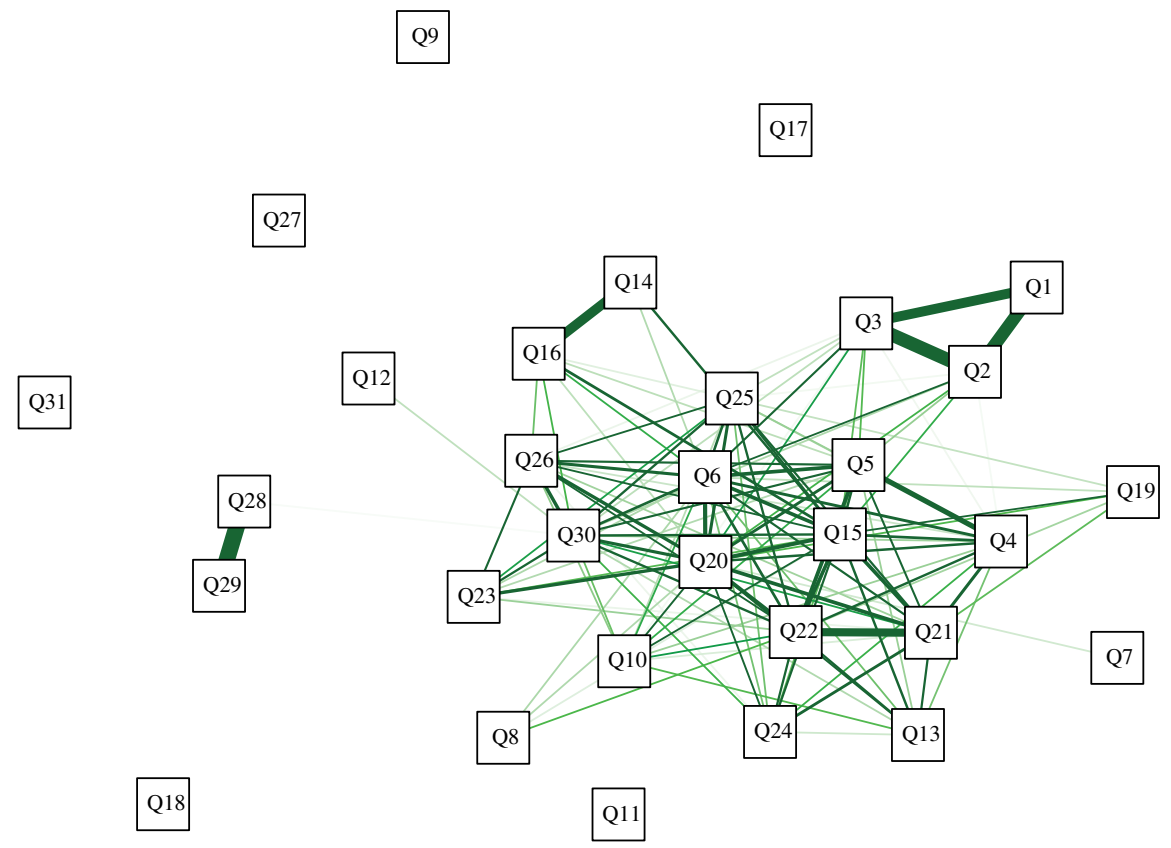

FIG. 2. Correlation matrix. Solid (green) lines represent positive correlations; dashed (red) lines negative correlations. Thicker lines represent larger correlations. 


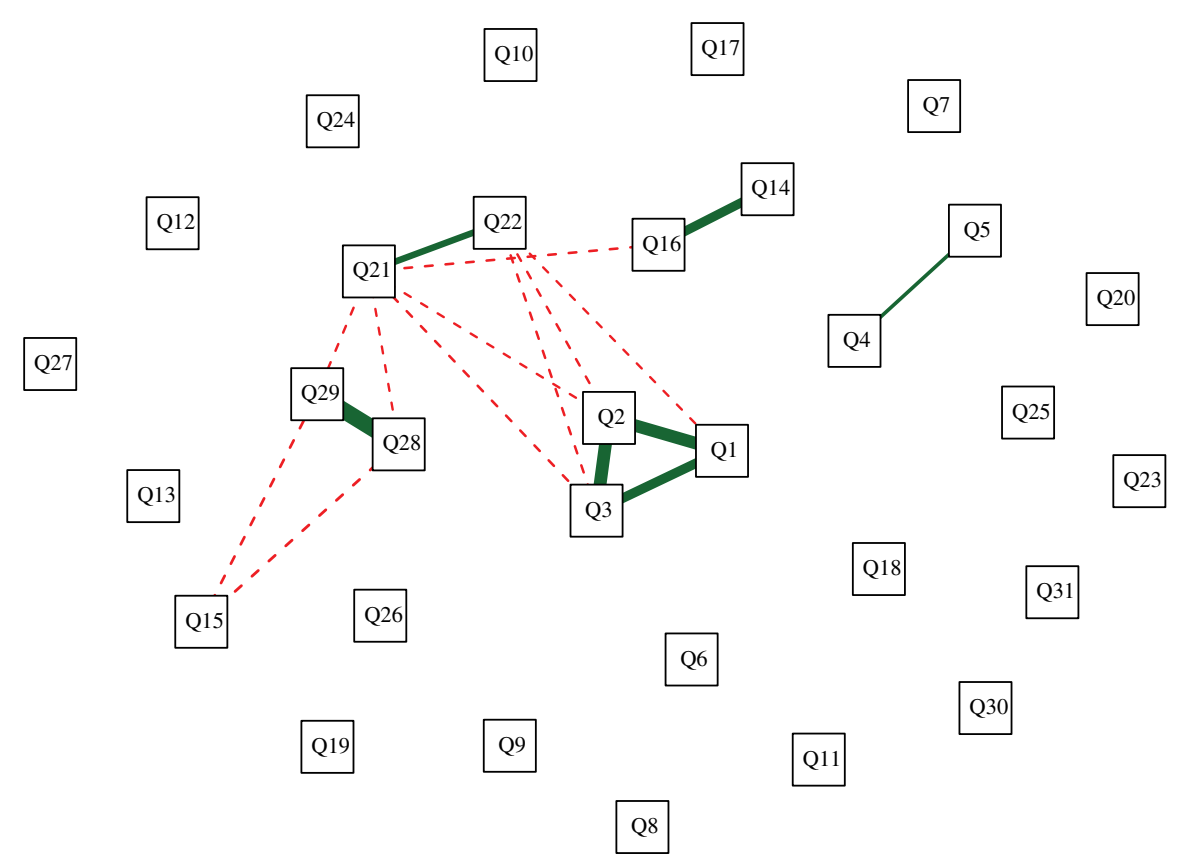

FIG. 3. Partial correlation matrix. Solid (green) lines represent positive correlations; dashed (red) lines negative correlations. Thicker lines represent larger correlations.

phrase level. Sentences and phrases representing the same fundamental reasoning process were grouped; these groups were called "principles." As in studies 1 to 3, the principles were then classified as laws (L) representing physical laws such as Gauss's law, definitions (DF) introducing a new quantity, and facts $(\mathrm{F})$ representing physical knowledge that was not as general as a law. From these primary principles, which define the core physical knowledge tested by the instrument, secondary principles were derived. The secondary principles included corollaries (C) and lemmas (LM). Corollaries are important secondary results of the laws, definitions, and facts. Qualitative statements in the solutions that interpreted laws, definitions, and corollaries were called lemmas. Some secondary principles were derived from primary principles that were not included in the expert solutions. These principles were inferred and

TABLE I. MIRT fit statistics for an exploratory factor analysis of the BEMA.

\begin{tabular}{lccccc}
\hline \hline Factors & AIC & BIC & RMSEA & TLI & CFI \\
\hline 1 & 332570 & 333015 & 0.07 & 0.80 & 0.80 \\
2 & 325941 & 326601 & 0.05 & 0.89 & 0.91 \\
3 & 321556 & 320413 & 0.02 & 0.95 & 0.96 \\
4 & 319343 & 320412 & 0.02 & 0.98 & 0.98 \\
5 & 318895 & 320158 & 0.02 & 0.99 & 0.99 \\
6 & 318657 & 320107 & 0.06 & 0.85 & 0.90 \\
7 & 318667 & 320296 & 0.06 & 0.84 & 0.91 \\
8 & 318872 & 320674 & 0.06 & 0.85 & 0.92 \\
9 & 318694 & 320713 & 0.06 & 0.84 & 0.92 \\
10 & 318694 & 320818 & 0.06 & 0.85 & 0.93 \\
\hline \hline
\end{tabular}

included in the model in Table III. Some of the principles in Table III are characterized with a bold font. These principles are those that are retained in the best-fitting principle model (M13) found through constrained MIRT. Finally, broad subtopics were introduced: mechanics, superposition, electrostatics, electric potential, magnetostatics, magnetic induction, and electric circuits.

Several principles in the electric circuits subcategory are secondary principles that could be derived from a primary principle. For example, C9 (current is the same throughout a series circuit) is derived from the law of conservation of charge. None of the expert solutions used these primary principles and it seemed unlikely that a student would use the primary principle. Such principles were not included in Table III and were not explored in the MIRT analysis.

Some BEMA items had multiple expert solution paths including items 4, 5, 7, 24, 25, and 31 (item 2 also had a secondary solution path, but was eliminated from the analysis because of blocking). In Table III, the principles necessary for secondary and tertiary solution paths are presented in parentheses with the solution path number within the parenthesis. For example, the first solution path of item 4 uses L5, L11, LM1, DF1, and LM2; the second solution path uses only $\mathrm{C} 2$. These different solution paths were explored using MIRT to determine which solution path was the most important to model student thinking.

Some principles were always used together. Borrowing the terminology of factor analysis, when a principle is used in an item it is said to "load" onto that item. In the BEMA, Faraday's law and the definition of magnetic flux are used together to solve items 28,29, and 31, but are not used in 
TABLE II. Factor structure for the five-factor model. Only loadings greater than 0.3 are shown. The factors are labeled FC1 to FC5.

\begin{tabular}{|c|c|c|c|c|c|}
\hline BEMA Item & $\mathrm{FC} 1$ & $\mathrm{FC} 2$ & FC3 & FC4 & FC5 \\
\hline 1 & & 0.92 & & & \\
\hline 2 & & 0.97 & & & \\
\hline 3 & & 0.90 & & & \\
\hline 4 & 0.46 & & & & \\
\hline 5 & 0.44 & & & & \\
\hline 6 & 0.36 & & & & 0.38 \\
\hline \multicolumn{6}{|l|}{7} \\
\hline \multicolumn{6}{|l|}{8} \\
\hline \multicolumn{6}{|l|}{9} \\
\hline 10 & & & & & 0.31 \\
\hline \multicolumn{6}{|l|}{11} \\
\hline \multicolumn{6}{|l|}{12} \\
\hline 13 & 0.40 & & & & \\
\hline 14 & & & & 0.87 & \\
\hline 15 & 0.44 & & & 0.40 & 0.36 \\
\hline 16 & & & & 0.88 & \\
\hline \multicolumn{6}{|l|}{17} \\
\hline \multicolumn{6}{|l|}{18} \\
\hline 19 & 0.31 & & & & \\
\hline 20 & 0.44 & & & & 0.43 \\
\hline 21 & 0.89 & & & & \\
\hline 22 & 0.79 & & & & \\
\hline 23 & & & & & 0.35 \\
\hline 24 & 0.40 & & & & \\
\hline 25 & 0.34 & & & & 0.34 \\
\hline 26 & 0.31 & & & & 0.42 \\
\hline \multicolumn{6}{|l|}{27} \\
\hline 28 & & & 0.97 & & \\
\hline 29 & & & 0.98 & & \\
\hline 30 & 0.39 & & & & 0.31 \\
\hline 31 & & & & & \\
\hline
\end{tabular}

other items. As such, MIRT cannot resolve them as a single principle. In the MIRT analysis, these principles were combined as a single principle labeled L9-DF7. Similarly, Gauss's law (L6) and the definition of electric flux (DF2) load together on item 18 as L6-DF2. Many other combined principles are shown in Table III.

Not all items were retained in the analysis. There are several problem blocks where multiple problems refer to a common physical system or refer to the same image. Studies 1 to 3 showed that blocked items can exhibit correlations unrelated to the physical reasoning needed to solve the item. Each item block was examined to determine if the items in the block were fairly independent. Items 2 and 3 depend on the response to item 1 and were removed from the analysis. Responses to items 4 and 5 do not depend on each other and were retained. Item 16 depends on the responses from items 14 and 15 and was removed. The responses to items 21 and 22 are fairly independent and were retained. Item 27 depends on the response from item 26 and was removed. The responses to items 28 and 29 are independent and were retained. The removed items still appear in Table III but were not included in the analysis.

Table III also indicates whether the principle was also tested by the CSEM to facilitate the comparison of the two instruments.

\section{Model transformation plan}

In a confirmatory analysis, the theoretical model is fit, then a series of theoretically motivated model transformations are performed to possibly improve the initial model fit. Table III represents the initial model and was fit first. Items 4, 5, 7, 24, 25, and 31 have multiple solution paths. For each of these items, the first solution path, shown in

TABLE III. Theoretical model tested by the BEMA. An $\times$ indicates that the principle is used in the CSEM.

\begin{tabular}{|c|c|c|c|c|}
\hline Label & Derived from & CSEM principle & BEMA No. & Principle \\
\hline \multicolumn{5}{|c|}{ Mechanics } \\
\hline L1 & & & 26,27 & Newton's 1st law. \\
\hline $\mathrm{L} 2$ & & $\times$ & & Newton's 2nd law. \\
\hline L3 & & $\times$ & $2(2)$ & Newton's 3rd law. \\
\hline C1 & L2 & $\times$ & 6,23 & $\begin{array}{l}\text { If a particle is turning in some direction, there is a force } \\
\text { in that direction. }\end{array}$ \\
\hline \multicolumn{5}{|c|}{ Electrostatics } \\
\hline L4 & & $\times$ & $1,2(1), 3,7(2)$ & Coulomb's law for the electric force $\left(\vec{F}=\frac{k q_{1} q_{2}}{r^{2}} \hat{r}\right)$. \\
\hline L5 & & $\times$ & $4(1), 5(1)$ & Coulomb's law for the electric field $\left(\vec{E}=\frac{k q}{r^{2}} \hat{r}\right)$ \\
\hline LM1 & L4 & $\times$ & $4(1), 5(1)$ & Opposite charges attract or likes repel. \\
\hline DF1 & & $\times$ & $4(1), 5(1), 6,26,27$ & Definition electric field $(\vec{F}=q \vec{E})$ \\
\hline LM2 & L5 & & $4(1), 5(1), 7(2)$ & Electric field weakens as distance increases. \\
\hline $\mathrm{C} 2$ & & & $4(2), 5(2)$ & Electric dipole field shape. \\
\hline $\mathbf{C 3}$ & $\mathrm{F} 1, \mathrm{LM} 2$ & & $7(1)$ & Charged object attracts a neutral object. \\
\hline $\mathrm{F} 1$ & & & $7(2)$ & An insulator polarizes in an external field. \\
\hline L6 & & & 18 & Gauss's law $\left(\oint_{S} \vec{E} \cdot \hat{n} d A=\frac{Q}{\epsilon_{0}}\right)$ \\
\hline
\end{tabular}


TABLE III. (Continued)

\begin{tabular}{|c|c|c|c|c|}
\hline Label & Derived from & CSEM principle & BEMA No. & Principle \\
\hline DF2 & & & 18 & Definition of electric flux $\left(\Phi=\int_{S} \vec{E} \cdot \hat{n} d A\right)$. \\
\hline F2 & & & 19 & Electric field is zero in a conductor. \\
\hline \multicolumn{5}{|c|}{ Electric potential } \\
\hline DF3 & & $x$ & 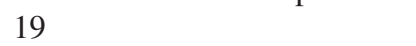 & Definition of electric potential $\left(\Delta V=\frac{W_{\mathrm{ext}}}{a}=-\int E d x\right)$. \\
\hline LM3 & DF3 & $\times$ & 14,16 & Electric field points to lower potential. ${ }^{q}$ \\
\hline LM4 & DF3 & & 14,16 & Potential difference in uniform field $(|\Delta V|=|E d|)$ \\
\hline LM5 & DF3 & & 15,16 & Potential difference is zero perpendicular to the field. \\
\hline LM6 & DF3 & & 16 & Total potential difference is the sum of $\Delta V$ over paths. \\
\hline \multicolumn{5}{|c|}{ Magnetostatics } \\
\hline L7 & & $x$ & $24(2), 25(2)$ & Biot-Savart law $\left(d \vec{B}=\frac{\mu_{0}}{4 \pi} \frac{I d \vec{\ell} \times \hat{r}}{r^{2}}\right)$. \\
\hline L15 & & & & Ampere's law $\left(\oint \vec{B} \cdot \vec{d} l \stackrel{4 \pi}{=} \mu_{0} I\right)$ \\
\hline LM7 & L15 & & $31(1)$ & Magnetic field is proportional to current. \\
\hline L8 & & $x$ & $23,25(2), 25(3), 26,27,30$ & Lorentz force $(\vec{F}=q \vec{v} \times \vec{B}$ or $d \vec{F}=I d \vec{\ell} \times \vec{B})$ \\
\hline LM8 & L8 & $\times$ & 20 & The magnetic force on a stationary charge is zero. \\
\hline LM9 & L7, L8, DF4 & $\times$ & $25(1)$ & Like currents attract or opposites repel. \\
\hline F3 & & & $21,22,24(3)$ & Magnetic dipole field shape. \\
\hline DF4 & & $\times$ & $23,24(2), 26,27,30$ & Right-hand rule for the cross product. \\
\hline DF5 & & $\times$ & 27 & Magnitude of the cross product $(|\vec{A} \times \vec{B}|=|\vec{A}||\vec{B}| \sin \theta)$. \\
\hline $\mathrm{C} 5$ & L7, DF4 & & $24(1), 25(3)$ & Right-hand rule for a wire. \\
\hline DF6 & & & $24(3)$ & Right-hand rule for a magnetic moment. \\
\hline C6 & L8, DF4 & & 30 & $\begin{array}{l}\text { Conductor moving in a magnetic field experiences a } \\
\text { potential difference. }\end{array}$ \\
\hline \multicolumn{5}{|c|}{ Induction } \\
\hline L9 & & $x$ & $28,29,31(1)$ & Faraday's law $\left(\mathrm{emf}=-\frac{d \Phi}{d t}\right)$ \\
\hline DF7 & & $\times$ & $28,29,31(1)$ & Definition of magnetic flux $\left(\Phi=\int_{S} \vec{B} \cdot \hat{n} d A\right)$. \\
\hline L10 & & & 28,29 & Lenz' law. \\
\hline C7 & L9, L10 & & 28,29 & Right-hand rule for changing flux. \\
\hline $\mathrm{C} 8$ & L9, L10 & & $31(2)$ & Mutual inductance $\left(\mathrm{emf}=-M \frac{d \Phi}{d t}\right)$. \\
\hline \multicolumn{5}{|c|}{ Superposition } \\
\hline L11 & & $x$ & $4(1), 5(1), 24$ & Electric and magnetic fields add as vectors. \\
\hline \multicolumn{5}{|c|}{ Electric circuits } \\
\hline F4 & & & 8,9 & $\begin{array}{l}\text { Battery produces current flowing from the }+ \text { terminal } \\
\text { through the circuit to the-terminal. }\end{array}$ \\
\hline DF8 & & & 9 & $\begin{array}{l}\text { Positive current is in the direction of flow of positive } \\
\text { charge or opposite the direction of flow of negative } \\
\text { charge. }\end{array}$ \\
\hline F5 & & & 10 & Ammeters have negligible resistance. \\
\hline C9 & & & 10 & Current same in series. \\
\hline F6 & & & 11 & Brighter light bulb indicates more current. \\
\hline L12 & & & $10,11,17$ & Ohm's law $(\Delta V=I R)$ \\
\hline C10 & & & 11 & Parallel elements have the same potential difference. \\
\hline C11 & & & 11 & Resistance adds for resistors in series. \\
\hline L13 & & & 12 & Ohm's law for the electric field $(\vec{J}=\sigma \vec{E})$. \\
\hline F7 & & & 17 & Complete circuit required for current flow. \\
\hline L14 & & & 17 & Kirchhoff's loop rule. \\
\hline DF9 & & & 13 & Definition of capacitance $\left(C=\frac{Q}{\Delta V}\right)$. \\
\hline F8 & & & 13 & $\mathrm{RC}$ circuits decay. \\
\hline
\end{tabular}

Table III with a "(1)," was fit in the initial model. For example, principle L4 is used in solution path 1 of item 2 which is shown as "2(1)" in Table III. The second solution path for each item was then fit, followed by the third solution path for items 24 and 25 . These model fits were compared with the original model and any model that was an improvement was retained.

The granularity of student knowledge was then explored to determine if the secondary principles were needed to understand student thinking. Models were constructed 
which removed the secondary principles - the lemmas (LM) and corollaries (C) - by replacing them with the primary principles from which they were derived. For example, LM1 (opposites attract or likes repel) can be derived from L4 (Coulomb's force law). To test whether LM1 was needed in addition to L4, all items that were set to load on LM1 in the initial model were set to load on L4 in the transformed model. This model was fit and fit statistics compared with the original model. This process was called "collapsing" LM1 into L4. Seven models were transformed in this way; C1 was collapsed into L1; LM1 was collapsed into L4; LM2 was collapsed into L5; LM3, LM4, LM5, and LM6 were collapsed into DF3; and LM8 was collapsed in L8. In the case of LM3, LM4, LM5, and LM6, any item loading onto one of the principles was set to load onto DF3. Two additional models were constructed which required a somewhat more complex transformation. The other two models were slightly more complex in that the secondary principle was not derived from a single primary principle but rather from several primary principles. In M15, LM9 was set to load onto L10, DF7, and DF4; in M16, C7 was set to load onto L9, L10, DF7, and DF4. These models were fit; models with improved fit were retained.

The definition of electric potential (DF3) loads onto item 19 with fact F2 (the electric field inside a conductor is zero). These two principles were not combined as a single principle in the MIRT analysis because during the third set of transformations LM3, LM4, LM5, and LM6 were all collapsed into DF3. Because of this transformation, F2 and DF3 no longer load on an item exclusively together, and so to maintain a nested model sequence they were not combined in the initial model.

The final set of transformations collapsed the principles and items onto the general topics of electricity and magnetism which form the divisions in Table III. These models are called "topical models." The first transformation, M17, collapsed each principle onto the general topics of electricity and magnetism: mechanics, electrostatics, electric potential, magnetostatics, magnetic induction, superposition, and electric circuits. To form M18, the principles involving mechanics and superposition were removed so as to include only topics specific to electricity and magnetism. This resulted in each item loading onto a single topic with the exception of item 26 which loaded onto both electrostatics and magnetostatics (a constant, uniform electric field and a constant, uniform magnetic field are both acting on a charged particle).

\section{Constrained MIRT}

In the exploratory work in Sec. III A, the MIRT discrimination matrix $\mathbf{a}_{\mathbf{j}}$ was allowed to take on any value. To apply MIRT as a confirmatory method, elements of the discrimination matrix which cannot theoretically be involved in solving an item are constrained to zero. For example, L1 (Newton's 1st law) is only used in items 26 and 27; $a_{j, L 1}$ was only allowed to be nonzero for items 26 and 27. This constraint means that abilities associated with the application of principles, not theoretically required for the solution of the item, do not influence the probability of answering the item correctly. In this way, the theoretical model in Table III is mapped onto the MIRT discrimination matrix. This analysis proceeds with the 27 -item instrument removing some of the blocked items. The reduced instrument contains items $1,4,5,6,7,8,9,10,11,12,13,14,15$, $17,18,19,20,21,12,13,14,25,26,28,29,30$, and 31 .

The transformation plan was carried out in panels: first testing each type of transformation independently to identify those which improved model fit, then combinations of the transformations which improved model fit were investigated. The results of carrying out the transformation plan are shown in Table IV. The "transformed model" column shows the model number of the model after the transformation has been applied to a prior model (the "original model"); M0 is the initial model which implements the model in Table III. The "transformation" column summarizes the transformation applied to the original model to form the transformed model. The fit statistics are then presented for the transformed model and the best fitting of the two models identified by the fit statistics and indicated in the "superior model" column.

The first set of transformations tried alternate solution paths identified in the expert solutions. Many of these alternate solutions produced superior models. For item 24, both solution paths 2 and 3 improved model fit with M4 producing superior fit; of these two transformed models, only M4 was used in further models. The next set of transformations tried combinations of the transformations in the first stage that produced superior models. All these combinations failed to improve on model M4. As such, the only modification to the initial model M0 that was retained was using the 3rd solution path to item 24. Item 24 asks about the magnetic field at the center of two parallel loops of wire; solution path 3 solved the item using the dipole moments of the loops.

The next set of transformations investigated whether the lemmas were required to model student thinking. Lemmas are qualitative principles derived from the generally quantitative laws and definitions. Studies 1 to 3 found lemmas were retained in the best-fitting model to a varying degree. In study 1 of the FCI, all lemmas were removed together which improved model fit. In studies 2 and 3, the lemmas were removed individually and some lemmas were retained in the best-fitting model. Lemmas play an important role in electromagnetism with LM1 (opposites attract or like repel) often used as part of the solution to both qualitative and quantitative problems. Only M13 was superior to M4; M13 combined lemmas LM3, LM4, LM5, and LM6 into DF3. DF3 is the definition of electric potential and the lemmas are different properties of electric potential such as the electric field points to lower potential (LM3) or the 
TABLE IV. Model transformation table. Each entry presents the result of modifying a prior model (the original model) with one of the planned transformations to produce a modified model (the transformed model). These two models are compared and the model with superior fit statistics identified (the superior model).

\begin{tabular}{|c|c|c|c|c|c|c|c|}
\hline Transformed model & Transformation & Original model & AIC & $\mathrm{BIC}$ & RMSEA & TLI & Superior model \\
\hline \multicolumn{8}{|c|}{ Full model } \\
\hline M0 & & $\ldots$ & 289783 & 290536 & 0.016 & 0.9870 .990 & $\cdots$ \\
\hline \multicolumn{8}{|c|}{ Explore alternate solution paths } \\
\hline M1 & Solution path 2 for items 4,5 & M0 & 289793 & 290496 & 0.016 & 0.9870 .990 & M0 \\
\hline M2 & Solution path 2 for item 7 & M0 & 289766 & 290533 & 0.016 & 0.9870 .990 & M2 \\
\hline M3 & Solution path 2 for item 24 & M0 & 289767 & 290528 & 0.016 & 0.9870 .990 & M3 \\
\hline M4 & Solution path 3 for item 24 & M0 & 289727 & 290488 & 0.016 & 0.9880 .991 & M4 \\
\hline M5 & Solution path 2 for item 25 & M0 & 289877 & 290645 & 0.016 & 0.9880 .990 & M0 \\
\hline M6 & Solution path 3 for item 25 & M0 & 289752 & 290513 & 0.015 & 0.9880 .991 & M6 \\
\hline M7 & Solution path 2 for item 31 & M0 & 289758 & 290505 & 0.015 & 0.9870 .990 & M7 \\
\hline \multicolumn{8}{|c|}{ Combine alternate solution path models } \\
\hline M8 & Combine $\mathrm{M} 2$ and $\mathrm{M} 4$ & M4 & 289721 & 290496 & 0.016 & 0.9880 .991 & M4 \\
\hline M9 & Combine M6 and M4 & M4 & 289860 & 290628 & 0.016 & 0.9880 .990 & M4 \\
\hline M10 & Combine M7 and M4 & M4 & 289759 & 290512 & 0.016 & 0.9880 .990 & M4 \\
\hline \multicolumn{8}{|c|}{ Collapse lemma into primary principles } \\
\hline M11 & Combine LM1 with L4 & M4 & 289746 & 290507 & 0.016 & 0.9880 .990 & M4 \\
\hline M12 & Combine LM2 with L5 & M4 & 289732 & 290486 & 0.016 & 0.9880 .990 & M4 \\
\hline M13 & Combine LM3, LM4, LM5, LM6 to DF3 & M4 & 289684 & 290445 & 0.015 & 0.9900 .992 & M13 \\
\hline M14 & Combine LM8 with L8 & M4 & 289739 & 290492 & 0.016 & 0.9870 .990 & M4 \\
\hline M15 & Combine LM9 with L7, L8, DF4 & M4 & 289826 & 290603 & 0.016 & 0.9880 .991 & M4 \\
\hline M16 & Combine C7 with L9, L10, DF7, DF4 & M4 & 289725 & 290500 & 0.016 & 0.9880 .991 & M4 \\
\hline \multicolumn{8}{|c|}{ Topical models } \\
\hline M17 & Collapse all principles into main topics & M13 & 289471 & 290117 & 0.015 & 0.9890 .991 & M17 \\
\hline M18 & Collapse all items into main topics & M17 & 289435 & 290024 & 0.016 & 0.9890 .990 & M18 \\
\hline
\end{tabular}

potential difference is zero perpendicular to the field (LM5). As such, most lemmas were important to modeling student reasoning about electromagnetism with only lemmas involving electric potential removed from the bestfitting model.

The final set of transformation tested more general models of student thinking using the general electromagnetic topics which form the divisions in Table III. In M17, the principles were set to load on the subtopic containing the principle; as such, some items loaded onto an electromagnetism subtopic and also onto the topics of mechanics and superposition. In M18, the subtopics of mechanics and superposition were removed and items were only loaded onto the electromagnetism subtopics. The fit statistics of M18 were superior to all other models. This result was diametrically opposite to that of studies 1 to 3 where the MIRT models involving the individual principles were superior to models using general topics. Possible reasons for this difference are explored as part of RQ3.

With M18 having superior fit statistics over more detailed models, one might consider whether the instrument is simply unidimensional with no substructure. A model containing only the $a_{0}$ discrimination is equivalent to the one-factor model in Table I. Comparison of the one-factor model and M18 shows that M18 is a substantially superior model with consistently superior fit statistics.

As such, M13 was the best-fitting model of student reasoning based on the granular model in Table III involving reasoning principles. The principles contained in this model are bolded in the table. M18 was the overall best fitting model. We note both M13 and M18 have exceptional RMSEA, CFI, and TLI, and as such, both can provide useful insights into student thinking. The difference in AIC and BIC likely results from the penalty these statistics place

TABLE V. Subscale scores for each topic. The mean \pm the standard deviation (SD) are shown. The mean calculates the average fraction of item in the subscale answered correctly by the students.

\begin{tabular}{lccccc}
\hline \hline & Electrostatics & Electric circuits & Electric potential & Magnetostatics & Magnetic induction \\
\hline Items & $1,4,5,6,7,26$ & $8,9,10,11,12,13,17$ & $14,15,19$ & $20,21,22,23,24,25,26,30$ & $28,29,31$ \\
Mean \pm SD & $0.59 \pm 0.24$ & $0.66 \pm 0.30$ & $0.48 \pm 0.21$ & $0.57 \pm 0.27$ & $0.22 \pm 0.30$ \\
Cronbach's $\alpha$ & 0.51 & 0.38 & 0.37 & 0.69 & 0.54 \\
\hline \hline
\end{tabular}


on the addition of parameters. The structure of M13 is further explored in Sec. III D, the structure of M18 in Sec. III C.

\section{Topical model}

The overall best-fitting model, M18, involved only the general electromagnetic topics which suggests these topics may be used as subscales, coherent measures of the topic. This model was called the "topical model." Table V shows the average fraction of students answering the items in the general topical groups (subscales) correctly. The electrostatics, magnetostatics, electric potential, and electric circuits subscales all have fairly similar averages differing by a maximum of $18 \%$. Table VI presents the item-level score (fraction of students answering the item correctly), $d_{j}$, general discrimination $a_{j 0}$, and subscale discrimination $a_{j k}^{s}$, where $j$ indexes the item and $k$ the subscale. Items within these subscales have a broad range of average item scores.
The parameter $d_{j}$ is related to the probability of answering the item correctly for students of average ability $(\vec{\theta}=0)$; items with larger $d_{j}$ are answered correctly with higher probability by students of average ability. The magnetic induction subscale has a much lower average score than the other subscales. It also contains two items, 28 and 29, with the lowest average score and the most negative $d_{j}$ of any items in the instrument. Items with negative $d_{j}$ are answered correctly less often than an item with average $d_{j}$.

In general, the range of discriminations $a_{j 0}$ was more narrow than the range of $d_{j}$. The discrimination is related to the slope of the probability curve with respect to $\theta$ at $\vec{a}_{j} \cdot \theta_{i}+d_{j}=0$, where the probability of selecting the correct response is 0.5 ; larger discriminations represent probability curves that are more steeply sloped at this point and a transition between a low probability of answering correctly and a high probability over a more narrow range of $\theta$. The item discriminates between low ability and high

TABLE VI. Best-fitting principle and topical MIRT models. The first column shows the item number (No.). Not all items of the BEMA were modeled. The discrimination for principle $k$ on item $j, a_{j k}$, is given by the number in parentheses following the principle label. The overall discrimination of item $j$ on a knowledge of electromagnetism is given by $a_{j 0}$. The difficulty of each item is related to $d_{j}$; items with larger positive $d_{j}$ are easier, items with more negative $d_{j}$, harder. The discrimination of the item on the subtopics of the topical model is given by $a_{j k}^{s}$.

\begin{tabular}{|c|c|c|c|c|c|c|c|c|}
\hline \multirow{2}{*}{$\begin{array}{l}\text { Item } \\
\text { No. }\end{array}$} & \multirow{2}{*}{$\begin{array}{l}\text { Item } \\
\text { score }\end{array}$} & \multicolumn{3}{|l|}{ Principle model (M13) } & \multicolumn{4}{|c|}{ Topical model (M18) } \\
\hline & & Principles & $a_{j 0}$ & $d_{j}$ & Topic & $a_{j k}^{s}$ & $a_{j 0}$ & $d_{j}$ \\
\hline 1 & 0.83 & $\mathrm{~L} 4(0.19)$ & 0.64 & 1.87 & Electrostatics & 0.08 & 0.58 & 1.70 \\
\hline 4 & 0.76 & DF1(0.21) LM1(0.24) LM2(0.11) L5(0.26) L11(0.19) & 1.35 & 2.00 & Electrostatics & 0.79 & 1.35 & 1.97 \\
\hline 5 & 0.54 & $\begin{array}{c}\mathrm{DF} 1(0.21) \operatorname{LM} 1(0.21) \mathrm{L} 5(0.37) \\
\operatorname{L11}(0.12)\end{array}$ & 1.37 & 0.28 & Electrostatics & 0.58 & 1.26 & 0.24 \\
\hline 6 & 0.57 & $\mathrm{C} 1(0.10) \mathrm{DF} 1(0.28)$ & 1.36 & 0.44 & Electrostatics & 0.15 & 1.24 & 0.38 \\
\hline 7 & 0.49 & $\mathrm{C} 3(0.18)$ & 0.72 & -0.03 & Electrostatics & -0.01 & 0.53 & -0.04 \\
\hline 8 & 0.76 & $\mathrm{~F} 4(0.08)$ & 0.72 & 1.32 & Electric circuits & 0.01 & 0.70 & 1.28 \\
\hline 9 & 0.30 & F4(0.10) DF8(0.19) & 0.13 & -0.95 & Electric circuits & 0.04 & 0.11 & -0.85 \\
\hline 10 & 0.60 & F5-C9(0.18) L12(0.22) & 0.97 & 0.55 & Electric circuits & 0.19 & 0.87 & 0.50 \\
\hline 11 & 0.43 & $\mathrm{~L} 12(0.16) \mathrm{F} 6-\mathrm{C} 10-\mathrm{C} 11(0.22)$ & 0.60 & -0.35 & Electric circuits & 0.10 & 0.53 & -0.31 \\
\hline 12 & 0.21 & $\mathrm{~L} 13(0.18)$ & 0.75 & -1.62 & Electric circuits & 0.09 & 0.69 & -1.51 \\
\hline 13 & 0.75 & DF9-F8(0.15) & 1.02 & 1.48 & Electric circuits & 0.10 & 0.93 & 1.38 \\
\hline 14 & 0.45 & DF3(0.28) & 0.62 & -0.22 & Electric potential & 0.29 & 0.63 & -0.23 \\
\hline 15 & 0.75 & DF3(0.50) & 1.80 & 1.99 & Electric potential & 0.48 & 1.77 & 1.94 \\
\hline 17 & 0.34 & L12(0.07) L14-F7(0.17) & 0.36 & -0.76 & Electric circuits & 0.10 & 0.33 & -0.71 \\
\hline 18 & 0.55 & L6-DF2(0.33) & 0.20 & 0.23 & Electrostatics & 0.01 & 0.18 & 0.20 \\
\hline 19 & 0.76 & F2(0.15) DF3(0.08) & 0.77 & 1.43 & Electric potential & 0.07 & 0.73 & 1.34 \\
\hline 20 & 0.58 & LM8(0.28) & 1.66 & 0.52 & Magnetostatics & 0.04 & 1.45 & 0.45 \\
\hline 21 & 0.84 & F3(1.43) & 2.55 & 4.25 & Magnetostatics & 1.71 & 2.82 & 4.77 \\
\hline 22 & 0.66 & $\mathrm{~F} 3(0.94)$ & 1.76 & 1.25 & Magnetostatics & 0.84 & 1.64 & 1.17 \\
\hline 23 & 0.49 & L8(0.09) DF4(0.08) C1(0.04) & 0.83 & -0.04 & Magnetostatics & -0.06 & 0.85 & -0.05 \\
\hline 24 & 0.68 & F3(0.11)L11(0.06) DF6(0.11) & 0.92 & 0.94 & Magnetostatics & 0.11 & 0.87 & 0.89 \\
\hline 25 & 0.56 & LM9 $(0.13)$ & 1.04 & 0.31 & Magnetostatics & 0.04 & 0.97 & 0.29 \\
\hline 26 & 0.39 & $\begin{array}{l}\text { DF1(0.18) L8(0.13) DF4(0.16) } \\
\text { L1(0.20) }\end{array}$ & 1.23 & -0.67 & $\begin{array}{l}\text { Electrostatics } \\
\text { Magnetostatics }\end{array}$ & $\begin{array}{l}-0.01 \\
-0.06\end{array}$ & 1.13 & -0.59 \\
\hline 28 & 0.18 & L9-DF7(2.14) C7-C8(3.21) & 2.25 & -7.51 & Magnetic induction & 5.53 & 2.44 & -7.95 \\
\hline 29 & 0.18 & L9-DF7(2.14) C7-C8(3.82) & 2.63 & -9.24 & Magnetic induction & 5.76 & 2.40 & -8.22 \\
\hline 30 & 0.40 & L8(0.18) DF4(0.11) C6(0.18) & 1.31 & -0.62 & Magnetostatics & -0.01 & 1.16 & -0.55 \\
\hline 31 & 0.30 & L9-DF7(0.07) LM7(0.12) & 0.29 & -0.92 & Magnetic induction & $(0.06)$ & 0.29 & -0.87 \\
\hline
\end{tabular}


ability students more strongly than lower discrimination items. All overall discriminations are positive, indicating items are generally well functioning. A negative discrimination would indicate the item was more likely to be answered correctly by lower ability students. The largest discriminations are associated with the two hardest items (items 28 and 29) involving magnetic induction and the easiest item (item 21) which asks about the direction of the magnetic field of a bar magnet. There are windowing effects relating $d_{j}$ and discrimination; an item with either very high or very low $d_{j}$ has a narrow range of $\theta$ to transition from low to high probability leading to high discrimination. Items 15 and 22 both have discriminations of about 1.5 with moderate item scores; item 15 asks about the electric potential difference along an equipotential and item 22 is blocked with item 21 and asks about the direction of the magnetic field of a bar magnet. Because of their moderate $d_{j}$ and high discrimination, these two items are probably the most effective for discriminating between high and low ability students. We note, in this context, and throughout this work, ability is narrowly defined as the facility to answer conceptual electromagnetism questions as presented in the BEMA.

The $d_{j}$ and overall discrimination of M13 was very similar to that of M18 and, therefore, the above discussion can be extended to this model as well.

The subscale discrimination, shown as $a_{j k}^{s}$ in Table VI, represents the amount the item discriminates on the subscale over its overall discrimination. Most subscale discriminations were fairly small; three of the largest discriminations were for items 15,28 , and 29 which also have large overall discrimination. Item 22, which is blocked with item 21 , also has a comparatively large discrimination. The only two other items that stand out are items 4 and 5 within the electrostatic subscale; the items are blocked and ask about the electric field direction at two points of an electric dipole. These two items do appear to more synthetically test for a knowledge of electrostatics than other items in the subscale. Item 26 requires a knowledge of both electrostatics and magnetostatics and has a subscale discrimination for both topics; both discriminations are small. This item largely discriminates on a students general facility with electromagnetism.

Characterizing the internal reliability or consistency of a subscale is a common problem in classical test theory. One of the most used statistics for internal reliability is Cronbach's $\alpha$ which is also presented in Table V [60]. The $\alpha$ values vary widely and none reach the threshold of 0.7 required for low stakes testing. As such, the subscales in Table $\mathrm{V}$ do not represent a coherent measurement of the subtopic, but rather represent the average of the student's knowledge on the individual items making up the subtopic. This is hardly surprising examining the broad set of reasoning represented by the principles in each subtopic.

\section{Principle model}

The principle model, M13, contains items requiring from 1 to 4 principles for their solution. For this discussion, principles that were combined because the MIRT model could not individually resolve them such as L9-DF7 were counted as a single principle. The overall $d_{j}$ and discrimination $a_{j 0}$ of each item was very similar in M13 and M18, and were discussed in Sec. IIIC. Table VI shows the principles used in M13 and the discrimination of each principle, in parentheses, as well as the overall discrimination $a_{j 0}$ of the item. Many principles had discriminations which were small compared to the overall discrimination; these items test a general facility with the material measured by the BEMA more strongly than the individual reasoning required by the principle. Some items had discrimination approximately commensurate with the overall discrimination: items 9, 18, 28, and 29. These items discriminate more strongly on the application of the principle than an overall facility with the material. The largest principle discriminations (items 20,21, 28, and 29) were generally associated with large overall discriminations. These items were discussed in the previous section. Very little stood out in the principle discriminations; most principles on the same item had similar discrimination and few items had one principle discrimination substantially different than the others.

Isomorphic items are items that are solved with the same process, items requiring the same principles for their solution. Item pairs $\{14,15\},\{21,22\}$, and $\{28,29\}$ are isomorphic. All are also part of item blocks complicating their statistical interpretation. Items 21 and 22 ask the student about the magnetic field at two different points around a bar magnet. Items 28 and 29 ask about the induced electric field direction at two points around a solenoid whose current is increasing. The similarity of items 14 and 15 are less clear. Item 14 involves the electric potential difference along an electric field line; item 15 involves the potential difference along an equipotential. Each item was initially coded as requiring different lemmas. All lemmas associated with the definition of electric potential (DF3) were collapsed into DF3 to form M13 which improved model fit. To determine if collapsing all electric potential items simultaneously obscured differences in student reasoning on items 14 and 15 , model M13 was modified to include LM5 (the electric potential difference is zero perpendicular to the field) as a separate principle. This transformation did not improve model fit. The students do not differentiate these two principles above their general difference in overall difficulty and discrimination.

\section{DISCUSSION}

\section{A. Research questions}

This study investigated three research questions; they will be discussed in the order proposed. The results of the 
individual analyses were discussed in the previous section as these analyses were introduced. This section summarizes and synthesizes the results of these analyses.

RQ1: What relations between BEMA items are identified by exploratory analyses? What do these relations imply for the interpretation of the results of applying the BEMA? Correlation analysis using the partial correlation correcting for overall instrument score (Fig. 3) showed that items within item blocks were correlated with each other above the average level of correlation expected of items testing a general knowledge of electromagnetism. The larger topical subscales tested by the instrument shown as subdivisions in Table III were not substantially correlated controlling for overall BEMA score as shown in Fig. 3. The blocked items stand out as the strongest correlations in the correlation matrix as well (Fig. 2); however, substantial positive correlations exist between many items. There is little evidence that items in the general subtopics in the topical model (M18) are generally more correlated with each other than with other items in the instrument in either the correlation or partial correlation matrix.

Exploratory factor analysis supported the conclusion that the blocked items represent the only statistically meaningful substructure of the instrument. Of the 5 factors in the best-fitting factor model (Table II), the highest loadings in four of the factors were items within the same item block. The fifth factor had no item with a large loading. Items from all subtopics except magnetic induction had similar, but small loadings, on factor 5 .

The prevalence of the blocked items in all the exploratory analysis strongly implies these items may be correlated more than would be the case if not blocked. This raises concerns about interpretation of the results of blocked items and suggests all items except the first in an item block be discarded. The grading rubric provided with the instrument at PhysPort [9] does suggest modified scoring rules for items 2 and 3 and items 28 and 29, all blocked items.

RQ2: What is the model of student knowledge measured by the BEMA identified by constrained MIRT? What insights can this model provide into the structure of the instrument? This work presented two models of the BEMA with excellent fit statistics: one featuring a detailed model of the instrument in terms of reasoning principles (M13) and one involving general electromagnetic subtopics (M18). Both of these models had similar and excellent fit statistics (RMSEA, CFI, and TLI). The topical model was better fitting measured by AIC and BIC probably because these measures penalize the additional parameters more strongly than RMSEA, CFI, and TLI. The Cronbach's $\alpha$ of the subtopics did not suggest they had strong internal consistency and the subtopics were not extracted as factors in factor analysis. As such, the principle model (M13), derived from a model of expert solutions of the instrument, may represent the best model of the instrument as a set of items that measure a broad set of fairly loosely related (in student thinking) pieces of electromagnetic reasoning.

The list of principles forming the initial model in Table III was extensive, larger than that of the FCI, FMCE, and CSEM in studies 1 to 3. The four models are compared in RQ3. Most principles, including secondary principles, were retained in the principle model (M13) indicating that student thinking about the material is composed of many disparate reasoning fragments. Many of these fragments were tested by single items making it difficult to explore student thinking in detail; for example, Gauss's law and the definition of electric flux are tested together by only a single item. There are a number of these combinations of principles that are only tested together which does not allow the instrument to determine if they are understood independently.

The shear breadth of principles and their variety, combined with the failure to find evidence that principles in the same subtopic are generally correlated above correlations through overall test score or to find subtopics as factors suggest that the overall design of the instrument may need refinement. An instrument with a more top down design around the five subtopics which focused on testing the most important principles within each subtopic well might provide instructors with a superior tool to manage their classes.

Classical test theory (CTT) suggests that items with either very high or very low items scores (called "difficulty" in CTT) or items with very low discrimination be considered problematic [60]. The item scores of items 1 , 21,28 , and 29 indicate that they may be problematic. Qualitatively, both IRT and CTT discriminations are similar measuring how well the items distinguishes between low and high performing students; however, they are not directly comparable quantitatively. As such, there is not a well established critical discrimination value for problematic MIRT items. Items with very small MIRT discriminations have fairly flat probability curves, so low and high ability students have similar probability of answer correctly. Items 9 and 18 have very small overall discrimination and should be investigated further to determine if they are functioning correctly.

RQ3: How are the best-fitting models of the BEMA, CSEM, FMCE, and FCI similar? How are they different? This work sought to understand the physical principles tested by the BEMA. It is the fourth of four papers using constrained MIRT to investigate some of the most widely applied physics conceptual instruments. To answer this research question, a comparison of the similarity and differences of the four instruments is provided. The BEMA is most topically related to the CSEM and specific comparisons to this instrument are made when appropriate. All four studies investigated three general dimensions: (i) the exploratory structure found by correlation analysis and factor analysis, (ii) the best-fitting principle model 
found by constrained MIRT and theoretically motivated modifications of an initial expert model, and (iii) a comparison of the best-fitting principle model to a more general model of the instrument (the topical model in the case of the BEMA).

Exploratory analyses of the FCI, FMCE, and BEMA proceeded first with a partial correlation analysis. All studies then employed exploratory factor analysis using MIRT. The best-fitting factor models were selected by examining fit statistics. The partial correlation analysis showed strong correlation between many blocked items; however, not all items within each item block were strongly partially correlated suggesting that, while important, blocking was not the only feature affecting the correlation structure. This pattern continued in the BEMA where item 15 was not strongly correlated with the other items in its block, items 14 and 16. Exploratory factor analysis of the four instruments yielded best-fitting factor models with from 5 to 9 factors: FCI (9), FMCE (5), CSEM-1 (9), CSEM-2 (8), and BEMA (5); study 3 presented two samples of CSEM data labeled CSEM-1 and CSEM-2. For all factor structures, the fit statistics did not clearly identify a single best-fitting model; different models were selected by different statistics. For all models, the factor structure had a strong relationship to the blocking structure of the instrument, but was not fully explained by the blocked structure. This effect was weaker in the CSEM with only 1 of the 3 item blocks consistently loading on the same factor in either sample. The strong effect of blocking was clearly evident for the BEMA; blocked items form the largest loadings on 4 of the 5 factors. The fifth factor includes many items across disparate topics, all with fairly low loadings. It is unclear what this factor actually measures. Blocked items explained only a subset of the 9 FCI factors; many of the other factors were related to isomorphic items which were not blocked. All FMCE factors were related to blocking, but all FMCE items except one are blocked. Like the FMCE, all isomorphic BEMA items $(\{14,15\},\{21,22\}$, and $\{28,29\})$ are also in item blocks, so the two effects cannot be separated.
The best-fitting principle models for each of the four conceptual inventories (FMCE, FCI, CSEM, and BEMA) can be compared to develop a greater understanding of the relationship of these instruments. This comparison may be valuable to practicing instructors trying to choose a conceptual instrument or to researchers comparing results of studies applying different instruments. Each study made a number of decisions about the inclusion of items in the analysis; therefore, the best-fitting principle models generally do not include all items while the initial theoretical models generally do include all items. Both the CSEM and BEMA contained combinations of principles where the combination always loaded on the same items; these combinations were coded as a single loading in MIRT. To compare instruments, these combinations contribute the number of principles in the combination toward the principle count. Two samples of the CSEM were analyzed producing slightly different best-fitting principle models. For comparison, CSEM sample 1 is used, because its principle model is the most similar to that of the BEMA. The models of the two CSEM samples differ only in the handling of the lemmas associated with electric potential.

Table VII presents a comparison of the BEMA and CSEM initial expert models which cover all items in the instruments. The principles are split into 3 groups: definitions and laws representing the most general coverage of the instrument, facts (representing specific knowledge needed to solve the instrument, and corollaries and lemmas representing qualitative and quantitative reasoning derived from the general principles needed to solve specific problems. The principles are also split between the subtopics introduced in Table III. Examining the definitions and lemmas column shows the BEMA in general covers most of the general physics covered by the CSEM, but the reverse is not true with the BEMA covering 10 additional principles. The number of principles covered by the other instrument is shown in parenthesis. Half of this difference involves the coverage of electric circuits. The difference in the $\mathrm{L}$ and DF principles between the instruments are generally localized to only a few items. For this discussion,

TABLE VII. Comparison of BEMA and CSEM. DF, L, R, C, and LM represent principles in each instrument. The number in parenthesis is the number of the principles also in the other instrument.

\begin{tabular}{|c|c|c|c|c|c|c|c|c|c|c|}
\hline \multirow[b]{2}{*}{ Subtopic } & \multicolumn{5}{|c|}{ BEMA } & \multicolumn{5}{|c|}{ CSEM } \\
\hline & Items & DF, L & $\mathrm{F}$ & $\mathrm{C}, \mathrm{LM}$ & Total & Items & DF, L & $\mathrm{F}$ & $\mathrm{C}, \mathrm{LM}$ & Total \\
\hline Mechanics & 0 & $3(2)$ & $0(0)$ & $1(1)$ & $4(3)$ & 0 & $3(2)$ & $0(0)$ & $2(1)$ & $5(3)$ \\
\hline Electrostatics & 8 & $5(3)$ & $2(0)$ & $4(1)$ & $11(4)$ & 14 & $5(3)$ & $1(0)$ & $4(1)$ & $10(4)$ \\
\hline Electric potential & 4 & $1(1)$ & $0(0)$ & $4(1)$ & $5(2)$ & 6 & $1(1)$ & $0(0)$ & $4(1)$ & $5(2)$ \\
\hline Magnetostatics & 9 & $6(4)$ & $1(0)$ & $5(2)$ & $12(6)$ & 9 & $4(4)$ & $1(0)$ & $2(2)$ & $7(6)$ \\
\hline Magnetic induction & 3 & $3(2)$ & $0(0)$ & $2(0)$ & $5(2)$ & 3 & $2(2)$ & $0(0)$ & $0(0)$ & $2(2)$ \\
\hline Superposition & 0 & $1(1)$ & $0(0)$ & $0(0)$ & $1(1)$ & 0 & $1(1)$ & $0(0)$ & $0(0)$ & $1(1)$ \\
\hline Electric circuits & 7 & $5(0)$ & $5(0)$ & $3(0)$ & $13(0)$ & 0 & $0(0)$ & $0(0)$ & $0(0)$ & $0(0)$ \\
\hline Total & 31 & $24(13)$ & $8(0)$ & $19(5)$ & $51(18)$ & 32 & $16(13)$ & $2(0)$ & $12(5)$ & $30(18)$ \\
\hline
\end{tabular}


TABLE VIII. Comparison of conceptual instruments. DF, L, R, F, C, LM, and RS represent principles in each instrument. Independent is abbreviated "ind" and principle "pcpl" when needed for spacing.

\begin{tabular}{|c|c|c|c|c|}
\hline & BEMA & CSEM-1 & FCI & FMCE \\
\hline Items analyzed & 27 & 25 & 20 & 43 \\
\hline $\mathrm{DF}, \mathrm{L}, \mathrm{R}$ & 18 & 16 & 9 & 5 \\
\hline $\mathrm{F}$ & 7 & 2 & 6 & 1 \\
\hline $\mathrm{C}, \mathrm{LM}, \mathrm{RS}$ & 13 & 6 & 4 & 2 \\
\hline Ind. principles & 38 & 24 & 19 & 8 \\
\hline Ind. pcpl. per item & 1.41 & 0.96 & 0.95 & 0.19 \\
\hline Items per ind. pcpl. & 0.71 & 1.05 & 1.05 & 5.38 \\
\hline 1 principle items & $10(37 \%)$ & $8(32 \%)$ & $4(20 \%)$ & $25(58 \%)$ \\
\hline 2 principle items & $5(29 \%)$ & $11(44 \%)$ & $7(35 \%)$ & $15(35 \%)$ \\
\hline 3 principle items & $6(22 \%)$ & $5(20 \%)$ & $7(35 \%)$ & $2(5 \%)$ \\
\hline 4 principle items & $5(19 \%)$ & $1(4 \%)$ & $1(5 \%)$ & $1(2 \%)$ \\
\hline 5 principle items & $1(4 \%)$ & $0(0 \%)$ & $1(5 \%)$ & $0(0 \%)$ \\
\hline Total principles & 63 & 49 & 48 & 65 \\
\hline Total pcpl. per item & 2.30 & 1.96 & 2.40 & 1.51 \\
\hline
\end{tabular}

differences in the use of mechanics is not considered. The CSEM includes two items involving the behavior of net charge on conductors and insulators, which require the law of conservation of charge. The CSEM also requires the student to read an electric field map which requires the definition of an electric field line. The BEMA contains a single Gauss's law item requiring both the application of Gauss's law and the definition of electric flux. The general coverage of magnetostatics is even more similar with the only difference found in the BEMA in one item applying the right-hand rule for magnetic moment along one solution path.

The specific coverage of the instruments, captured in the number of F, C, and LM principles, is fairly different. These principles involve the less general patterns of reasoning required to solve specific individual items. The majority of these types of principles are not shared between the instruments, only 5 of the 39 principles are shared. As such, while the general coverage of the instruments is similar (except for electric circuits), the specific coverage is quite different. Many more of these specific principles were identified in the BEMA; the CSEM covers electricity and magnetism at a somewhat more general level. This has important implications for the generalizability of BEMA or CSEM results because specific pedagogical choices can affect the detailed coverage of a class, as well as its general coverage.

One of the benefits of building a detailed model of an instrument such as that in Table III is the facilitation of new qualitative and quantitative comparisons between instruments. The general complexity of items in the instrument can be characterized by the average number of reasoning steps per item. The degree to which the instrument measures a piece of reasoning with multiple items (providing generally higher reliability) can be characterized by the number of items per reasoning step.
Table VIII presents a general comparison of the bestfitting principle models of all four instruments. The principle models did not fit all items (except in the FMCE), but do allow a more detailed comparison of the instruments on the items fit. The models of the FCI and FMCE involved two additional types of principles not found in the CSEM or BEMA: results (R) such as the threedimensional kinetic equations for motion under a constant force and reasoning steps (RS) such as reading a graph. Table VIII presents two measures of overall instrument length: the independent principles representing the number of unique principles needed to solve instrument and the total principles representing the number of reasoning steps required to solve the instrument. Each independent principle may be required to solve multiple items and thus be counted multiple times in the total principles. As above, the BEMA involved applying more independent principles (an independent principle represents one of the rows in Table III) than the CSEM, approximately $40 \%$ more independent principles per item. As such, a greater variety of physical knowledge is needed to solve each item. A larger fraction of the BEMA items were fairly complex requiring four or five principles for their solution. These two differences led to generally more complex items requiring 2.3 principles per item on average for the BEMA in comparison to 2.0 principles per item in the CSEM. As such, the BEMA generally involves applying longer, more complex, patterns of reasoning than the CSEM. This observation also implies that each principle in the BEMA is not as thoroughly measured as in the other instruments with only 0.71 items measuring each independent principle. The FMCE strongly stands out on this metric with each independent principle in the FMCE measured on average by over 5 items.

All four studies paid particular attention to the role of lemmas in the models. The other types of principles 
represent standard content that might be present in most textbooks; lemmas represent qualitative interpretations of these principles. All studies found that experts used many lemmas in their solutions; however, it was unclear whether these principles were needed to model student thinking. In study 1 , all lemmas were removed simultaneously which improved model fit. In the studies of the FMCE and CSEM, lemmas were removed in groups, as they were in the present study. The best-fitting model for the FMCE, CSEM, and BEMA all contained some, but not all, of the lemmas in the initial expert model. The lemmas remaining in the FMCE involved motion opposite the direction of acceleration and are associated with a type of problem particularly difficult for students. Many of the lemmas identified for the electricity and magnetism instruments represent principles central to solving qualitative (and quantitative) items such as "opposites attract-likes repel." As such, it would have been surprising if these principles were not found to be part of the model of student reasoning.

All studies explored a model more general than the best-fitting principle model. For the FCI, study 1 fit a decomposition of the items of the instruments into topics that was proposed with the original publication of the instrument [61]. The principle model improved AIC by 448 and BIC by 226 over the topical model, very strong changes. Study 3 fit a model similar to the best-fitting principle model of the current study (excluding the electric circuits topic); for both samples, the topical model had worse model fit than all of the principle models. For one of the samples, the best-fitting topical model did not meet the requirements of acceptable model fit [59]. The FMCE uses fewer principles and repeats the principles more often than the other instruments. As such, rather than proposing general topical principles, Study 2 grouped FMCE items into subscales. Confirmatory factor analysis was then performed to determine if this model fit the instrument well; it did not $(\mathrm{CFI}=0.80$, TLI $=0.79$, and RMSEA $=0.080$ ). So for the FCI, FMCE, and the CSEM, the more general topical model was substantially less well fitting than the best-fitting principle model. The results for the BEMA were different; the topical model was better fitting than the best-fitting principle model with difference in AIC and BIC similar to those observed for the FCI and larger than the differences observed for either CSEM sample. For context, the difference in the two model's AIC was 249, this means the topical model was $e^{249 / 2}$ times more probable than the principle model. The reason for this difference is unclear; perhaps the larger number of principles and the fairly weak interconnections of principles within items generates an instrument which is more a measurement of general topics than specific information within the topics. The BEMA topical model had similar RMSEA, CFI, and TLI to the principle model. Correlation and factor analysis also did not support the view of the instrument measuring independent subtopics; therefore, the best-fitting principle model may be a better general model of the knowledge measured by the BEMA.

\section{B. Synthesis}

Through the four studies applying constrained MIRT, some important themes have emerged. We attempt to encapsulate those themes in this section.

\section{The general quality of the initial expert model}

The studies of the FMCE, CSEM, and BEMA reported CFI, TLI, and RMSEA for each stage of the model transformation process. In general, the initial expert model had excellent fit statistics. These were improved only slightly through the transformation process. We revisited the models used for the FCI and a similar pattern of excellent fit throughout the transformation process was observed. As such, the initial expert models derived from observations of expert solutions were very good models of the material and could be constructed without the need to collect large datasets and without the application of MIRT. This observation opens the possibility of developing similarly detailed models of an entire domain such as introductory mechanics or electricity and magnetism. These models would allow one to quantitatively express the relationship between the conceptual instruments and the domain they profess to measure. The decisions about item selection and topical coverage used to construct the instruments could be evaluated by the PER community within this framework. Such a framework could also serve to allow more detailed description of instructional innovations by providing a mechanism to specify in detail any changes in topical coverage resulting from the innovation.

\section{The negative effect of item blocking}

All studies found that blocked items dominated both the partial correlation and exploratory factor structures. In some instruments, such as the FMCE and the CSEM, blocked items were often isomorphic. This was not the case in the FCI which strongly suggests that blocked items have correlations and other statistical properties that are the result of blocking, not the physical constructs the items were intended to measure. This and other work strongly suggests that item blocking should be discontinued in future instruments. It also suggests that exploratory factor structures extracted from instruments with item blocks including the FCI, FMCE, CSEM, and BEMA are strongly related to these blocks and is not a general measure of the substructure of student reasoning on the topic; conclusions drawn from these analyses should be interpreted with care. 


\section{The granularity of the knowledge measured by the instruments}

In the three studies that removed lemmas independently, the best-fitting models contained some lemmas and corollaries, very specific reasoning pieces. Further, in the studies of the FCI, FMCE, and the CSEM a general topical model was not as well fitting as the model involving a decomposition into principles. The topical model was better fitting for the BEMA by AIC and BIC, but had very similar (and sometimes weaker) RMSEA, CFI, and TLI than the bestfitting principle model. There was very little evidence in the correlation or factor analysis to suggest the items within the subtopics were more related with themselves than with items in other subtopics. As such, all of these popular instruments measure a detailed set of reasoning skills as opposed to a general construct such as "Newtonian thinking." This is supported by the analysis of the FMCE and the BEMA which found poor subscale internal consistency as measured by Cronbach's $\alpha$. This has important implications for the general interpretation of the results of applying the instruments; the instruments may be susceptible to small changes in the coverage or focus of the courses studied.

\section{The general dissimilarity of the instruments}

The constrained MIRT models produced a very detailed picture of the four conceptual instruments. While the FCI and FMCE, as well as the CSEM and BEMA, cover the same general topics (Newtonian mechanics or electromagnetism), the pairs of instruments were quite different through the detailed lens of MIRT. The quantitative differences are explored in the discussion of RQ3; the qualitative differences are self-evident through a comparison of the expert models. As such, comparing studies using different instruments should be done with care and should consider how the detailed differences of the instruments might interact with the student population or any pedagogical differences between treatments.

\section{Future work}

All four MIRT studies identified the blocking of items as a potential problem, generating correlations between items not related to the physical reasoning needed to solve the items. Network analytic studies have also identified items where connections between correct and incorrect responses suggest students may be answering correctly for incorrect reasons $[26,27,62]$. Multiple authors have suggested alternate scoring rubrics for some of these instruments in response to these and other problems $[9,27,63,64]$. Many classical test theory and item response theory studies have identified items within these instruments with performance outside the suggested range for good psychometric functioning $[17,18,44,45]$. Substantial biases have also been identified in some of the instruments [17]. With the accumulation of evidence that these instruments at the very least should be revisited and revised, a model of a revised instrument in terms of principles grounded in a more general model of the domain measured could provide basis for a discussion within the research community of what should be assessed in introductory physics leading to a new generation of conceptual instruments.

\section{LIMITATIONS}

This work was performed using data drawn for a single institution. The models should be tested with additional student populations to determine if the conclusions are general.

\section{CONCLUSIONS}

This study investigated the structure of the BEMA using correlation analysis and exploratory factor analysis and, then, explored the models of student knowledge tested by the BEMA using constrained MIRT. Correlation analysis revealed that items within item blocks account for nearly all of the substructure of the instrument. Exploratory factor analysis identified a five-factor model as having the best fit. The highest loadings in four of the five factors were items in the same item block, consistent with the correlation analysis. Two models of student knowledge were presented; one involved 28 detailed reasoning principles (M13) and the other contained five general electromagnetic subtopics (M18). Both models had excellent fit statistics. The five topics in M18 were investigated as subscales; however, none of the subscales had a Cronbach's $\alpha$ of 0.7 suggested for low-stakes testing. As such, the model of student knowledge tested by the BEMA consists of a broad collection of loosely related reasoning pieces.

The best-fitting principle models of the FCI, FMCE, and CSEM had fewer principles than that of the BEMA. The best-fitting principle model of the BEMA also required more lemmas and corollaries than any of the other instruments' models. The coverage differences between the CSEM and BEMA were largely the result of the coverage of electric circuits in the BEMA and differences in the coverage of electrostatics. Quantitative comparison of the four conceptual instruments investigated using constrained MIRT identified substantial differences in terms of the number of principles and the number of principles per item. As such, while related, the FCI and the FMCE as well as the CSEM and the BEMA measure their conceptual domains with different coverage and with items with different intellectual complexity.

\section{ACKNOWLEDGMENTS}

We thank Steven Pollock for the collection and curation of this exceptional dataset and his helpful commentary. 
[1] R. Chabay and B. Sherwood, Qualitative understanding and retention, AAPT Announcer 27, 96 (1997).

[2] The BEMA itself was never published in an archival journal. Early references to the instrument use Chabay and Sherwood (1997) (Ref. [1]) to cite the instrument. This issue of the AAPT Announcer is not available electronically. The citation references the program to the Summer 1997 American Association of Physics Teachers meeting then published in the Announcer. The page referenced contains Chabay and Sherwood's contributed talk abstracts about research applying the instrument. Interestingly, Maloney, O'Kuma, Van Heuvelen, and Hieggelke discussed challenges to developing an electricity and magnetism instrument in the same session which lead to the CSEM.

[3] D. P. Maloney, T. L. O'Kuma, C. Hieggelke, and A. Van Huevelen, Surveying students' conceptual knowledge of electricity and magnetism, Phys. Ed. Res., Am. J. Phys. 69, S12 (2001).

[4] I. A. Halloun and D. Hestenes, The initial knowledge state of college physics students, Am. J. Phys. 53, 1043 (1985).

[5] D. Hestenes, M. Wells, and G. Swackhamer, Force Concept Inventory, Phys. Teach. 30, 141 (1992).

[6] R. R. Hake, Interactive-engagement versus traditional methods: A six-thousand-student survey of mechanics test data for introductory physics courses, Am. J. Phys. 66, 64 (1998).

[7] R. K. Thornton and D. R. Sokoloff, Assessing student learning of Newton's laws: The Force and Motion Conceptual Evaluation and the evaluation of active learning laboratory and lecture curricula, Am. J. Phys. 66, 338 (1998).

[8] J. L. Docktor and J. P. Mestre, Synthesis of disciplinebased education research in physics, Phys. Rev. Phys. Educ. Res. 10, 020119 (2014).

[9] Physport, https://www.physport.org. Accessed 8/8/2017.

[10] S. DeVore, J. Stewart, and G. Stewart, Examining the effects of testwiseness in conceptual physics evaluations, Phys. Rev. Phys. Educ. Res. 12, 020138 (2016).

[11] J. Stewart, C. Zabriskie, S. DeVore, and G. Stewart, Multidimensional item response theory and the Force Concept Inventory, Phys. Rev. Phys. Educ. Res. 14, 010137 (2018).

[12] C. Zabriskie and J. Stewart, Multidimensional item response theory and the Conceptual Survey of Electricity and Magnetism, Phys. Rev. Phys. Educ. Res. 15, 020107 (2019).

[13] J. Yang, C. Zabriskie, and J. Stewart, Multidimensional item response theory and the Force and Motion Conceptual Evaluation, Phys. Rev. Phys. Educ. Res. 15, 020141 (2019).

[14] M. Planinic, L. Ivanjek, and A. Susac, Rasch model based analysis of the Force Concept Inventory, Phys. Rev. Phys. Educ. Res. 6, 010103 (2010).

[15] L. Ding, Applying Rasch theory to evaluate the construct validity of brief electricity and magnetism assessment, AIP Conf. Proc. 1413, 175 (2012).

[16] L. Ding, Seeking missing pieces in science concept assessments: Reevaluating the Brief Electricity and Magnetism Assessment through Rasch analysis, Phys. Rev. Phys. Educ. Res. 10, 010105 (2014).
[17] A. Traxler, R. Henderson, J. Stewart, G. Stewart, A. Papak, and R. Lindell, Gender fairness within the Force Concept Inventory, Phys. Rev. Phys. Educ. Res. 14, 010103 (2018).

[18] R. Henderson, P. Miller, J. Stewart, A. Traxler, and R. Lindell, Item-level gender fairness in the Force and Motion Conceptual Evaluation and the Conceptual Survey of Electricity and Magnetism, Phys. Rev. Phys. Educ. Res. 14, 020103 (2018).

[19] Y. Xiao, J. C. Fritchman, J. Y. Bao, Y. Nie, J. Han, J. Xiong, H. Xiao, and L. Bao, Linking and comparing short and fulllength concept inventories of electricity and magnetism using item response theory, Phys. Rev. Phys. Educ. Res. 15, 020149 (2019).

[20] P. Eaton, K. Johnson, B. Frank, and S. Willoughby, Classical test theory and item response theory comparison of the Brief Electricity and Magnetism Assessment and the Conceptual Survey of Electricity and Magnetism, Phys. Rev. Phys. Educ. Res. 15, 010102 (2019).

[21] J. Wang and L. Bao, Analyzing Force Concept Inventory with item response theory, Am. J. Phys. 78, 1064 (2010).

[22] S. Youngsuk and B. Daniel, Nested logit models for multiple-choice item response data, Psychometrika 75, 454 (2010).

[23] J. Stewart, B. Drury, J. Wells, A. Adair, R. Henderson, Y. Ma, A. Pérez-Lemonche, and D. Pritchard, Examining the relation of correct knowledge and misconceptions using the nominal response model, Phys. Rev. Phys. Educ. Res. 17, 010122 (2021).

[24] T. F. Scott and D. Schumayer, Students' proficiency scores within multitrait item response theory, Phys. Rev. Phys. Educ. Res. 11, 020134 (2015).

[25] J. Wells, R. Henderson, J. Stewart, G. Stewart, J. Yang, and A. Traxler, Exploring the structure of misconceptions in the Force Concept Inventory with modified module analysis, Phys. Rev. Phys. Educ. Res. 15, 020122 (2019).

[26] J. Wells, R. Henderson, A. Traxler, P. Miller, and J. Stewart, Exploring the structure of misconceptions in the Force and Motion Conceptual Evaluation with modified module analysis, Phys. Rev. Phys. Educ. Res. 16, 010121 (2020).

[27] C. Wheatley, J. Wells, R. Henderson, and J. Stewart, Applying module analysis to the Conceptual Survey of Electricity and Magnetism, Phys. Rev. Phys. Educ. Res. 17, 010102 (2021).

[28] S. J. Pollock, Longitudinal study of student conceptual understanding in electricity and magnetism, Phys. Rev. Phys. Educ. Res. 5, 020110 (2009).

[29] M. A. Kohlmyer, M. D. Caballero, R. Catrambone, R. W. Chabay, L. Ding, M. P. Haugan, M. J. Marr, B. D. Sherwood, and M. F. Schatz, Tale of two curricula: The performance of 2000 students in introductory electromagnetism, Phys. Rev. Phys. Educ. Res. 5, 020105 (2009).

[30] M. W. McColgan, R. A. Finn, D. L. Broder, and G. E. Hassel, Assessing students' conceptual knowledge of electricity and magnetism, Phys. Rev. Phys. Educ. Res. 13, 020121 (2017).

[31] L. Ding, R. Chabay, B. Sherwood, and R. Beichner, Evaluating an electricity and magnetism assessment tool: Brief Electricity and Magnetism Assessment, Phys. Rev. Phys. Educ. Res. 2, 010105 (2006). 
[32] L. Ding and R. Beichner, Approaches to data analysis of multiple-choice questions, Phys. Rev. Phys. Educ. Res. 5, 020103 (2009).

[33] R. W. Chabay and B. A. Sherwood, Matter and Interactions (John Wiley \& Sons, Hoboken, NJ, 2015).

[34] S. J. Pollock, Comparing student learning with multiple research-based conceptual surveys: CSEM and BEMA, AIP Conf. Proc. 1064, 171 (2008).

[35] P. Eaton, B. Frank, K. Johnson, and S. Willoughby, Comparing exploratory factor models of the Brief Electricity and Magnetism Assessment and the Conceptual Survey of Electricity and Magnetism, Phys. Rev. Phys. Educ. Res. 15, 020133 (2019).

[36] A. Newell and H. A. Simon, Human Problem Solving (Prentice-Hall, Englewood Cliffs, NJ, 1972).

[37] S. Ohlsson, The problems with problem solving: Reflections on the rise, current status, and possible future of a cognitive research paradigm, J. Prob. Solving 5, 7 (2012).

[38] J. Larkin, J. McDermott, D. P. Simon, and H. A. Simon, Expert and novice performance in solving physics problems, Science 208, 1335 (1980).

[39] J. H. Larkin, J. McDermott, D. P. Simon, and H. A. Simon, Models of competence in solving physics problems, Cogn. Sci. 4, 317 (1980).

[40] F. Reif and J. I. Heller, Knowledge structure and problem solving in physics, Educ. Psychol. 17, 102 (1982).

[41] U.S. News \& World Report: Education, https://premium .usnews.com/best-colleges. Accessed 4/30/2017.

[42] L. Chen, J. Han, J. Wang, and Y. Tu, Comparisons of Item Response Theory algorithms on Force Concept Inventory, Res. Edu. As. Learn. 2, 26 (2011).

[43] S. Osborn Popp, D. Meltzer, and M. C. Megowan-Romanowicz, Is the Force Concept Inventory biased? Investigating differential item functioning on a test of conceptual learning in physics, in 2011 American Educational Research Association Conference (American Education Research Association, Washington, DC, 2011).

[44] J. Han, L. Bao, L. Chen, T. Cai, Y. Pi, S. Zhou, Y. Tu, and K. Koenig, Dividing the Force Concept Inventory into two equivalent half-length tests, Phys. Rev. Phys. Educ. Res. 11, 010112 (2015).

[45] P. Eaton, K. Johnson, and S. Willoughby, Generating a growth-oriented partial credit grading model for the Force Concept Inventory, Phys. Rev. Phys. Educ. Res. 15, 020151 (2019).

[46] P. Eaton and S. Willoughby, Identifying a preinstruction to postinstruction factor model for the Force Concept Inventory within a multitrait item response theory framework, Phys. Rev. Phys. Educ. Res. 16, 010106 (2020).
[47] T. I. Smith, K. J. Louis, B. J. Ricci IV, and N. Bendjilali, Quantitatively ranking incorrect responses to multiplechoice questions using item response theory, Phys. Rev. Phys. Educ. Res. 16, 010107 (2020).

[48] T. F. Scott, D. Schumayer, and A. R. Gray, Exploratory factor analysis of a Force Concept Inventory data set, Phys. Rev. Phys. Educ. Res. 8, 020105 (2012).

[49] L. J. Cronbach and P.E. Meehl, Construct validity in psychological tests, Psychol. Bull. 52, 281 (1955).

[50] L. A. Clark and D. Watson, Constructing validity: Basic issues in objective scale development, Psychol. Assess. 7, 309 (1995).

[51] R. P. Chalmers, mirt: A multidimensional item response theory package for the R environment, J. Stat. Softw. 48, 1 (2012).

[52] R Core Team, R: A Language and Environment for Statistical Computing (R Foundation for Statistical Computing, Vienna, Austria 2017).

[53] L. Cai, A two-tier full-information item factor analysis model with applications, Psychometrika 75, 581 (2010).

[54] A. E. Raftery, Bayesian model selection in social research, Sociol. Methodol. 25, 111 (1995).

[55] A. Maydeu-Olivares and H. Joe, Limited information goodness-of-fit testing in multidimensional contingency tables, Psychometrika 71, 713 (2006).

[56] A. Maydeu-Olivares, Goodness-of-fit assessment of item response theory models, Measurement 11, 71 (2013).

[57] R. E. Schumacker and R. G. Lomax, A Beginner's Guide to Structural Equation (Routledge, New York, 2016).

[58] R. B. Kline, Principles and Practices of Structural Equation Modeling, 4th edition (Guilford Publications, New York, 2016).

[59] L. Hu and P. M. Bentler, Cutoff criteria for fit indexes in covariance structure analysis: Conventional criteria versus new alternatives, Struct. Equ. Modeling 6, 1 (1999).

[60] L. Crocker and J. Algina, Introduction to Classical and Modern Test Theory (Holt, Rinehart, and Winston, Mason, $\mathrm{OH}, 1986)$.

[61] D. Hestenes, M. Wells, and G. Swackhamer, Force Concept Inventory, Phys. Teach. 30, 141 (1992).

[62] J. Yang, J. Wells, R. Henderson, E. Christman, G. Stewart, and J. Stewart, Extending modified module analysis to include correct responses: Analysis of the Force Concept Inventory, Phys. Rev. Phys. Educ. Res. 16, 010124 (2020).

[63] R. C. Hudson and F. Munley, Re-score the Force Concept Inventory!, Phys. Teach. 34, 261 (1996).

[64] R. K. Thornton, D. Kuhl, K. Cummings, and J. Marx, Comparing the Force and Motion Conceptual Evaluation and the Force Concept Inventory, Phys. Rev. Phys. Educ. Res. 5, 010105 (2009). 